\title{
Low Power Wide Area Networks: An Overview
}

\author{
Usman Raza, Parag Kulkarni, and Mahesh Sooriyabandara
}

\begin{abstract}
Low Power Wide Area (LPWA) networks are attracting a lot of attention primarily because of their ability to offer affordable connectivity to the low-power devices distributed over very large geographical areas. In realizing the vision of the Internet of Things (IoT), LPWA technologies complement and sometimes supersede the conventional cellular and short range wireless technologies in performance for various emerging smart city and machine-to-machine (M2M) applications. This review paper presents the design goals and the techniques, which different LPWA technologies exploit to offer wide-area coverage to lowpower devices at the expense of low data rates. We survey several emerging LPWA technologies and the standardization activities carried out by different standards development organizations (e.g., IEEE, IETF, 3GPP, ETSI) as well as the industrial consortia built around individual LPWA technologies (e.g., LoRa ${ }^{\mathrm{TM}}$ Alliance, WEIGHTLESS-SIG, and DASH7 Alliance). We further note that LPWA technologies adopt similar approaches, thus sharing similar limitations and challenges. This paper expands on these research challenges and identifies potential directions to address them. While the proprietary LPWA technologies are already hitting the market with large nationwide roll-outs, this paper encourages an active engagement of the research community in solving problems that will shape the connectivity of tens of billions of devices in the next decade.
\end{abstract}

Index Terms-Internet of Things, IoT, Low Power Wide Area, LPWA, LPWAN, Machine-to-Machine Communication, Cellular

\section{INTRODUCTION}

$\mathbf{T}$ HE Internet of Things (IoT) promises to revolutionize the way we live and work. It could help us in overcoming the top global challenges due to population explosion, energy crisis, resource depletion, and environmental pollution. To realize this vision, things need to sense their environment, share this information among themselves as well as with humans to enable intelligent decision making for positively affecting our entire ecosystem. Due to this promise, an interest in IoT is phenomenal. Multiple independent studies have forecasted a rampant growth in volume and revenue of IoT and Machine-to-Machine (M2M) industry in the next ten years. Number of connected M2M devices and consumer electronics will surpass the number of human subscribers using mobile phones, personal computers, laptops and tablets by 2020 [1]. Moving forward, by 2024, the overall IoT industry is expected to generate a revenue of 4.3 trillion dollars [2] across different sectors such as device manufacturing, connectivity, and other value added services. Recent improvements in cheap sensor and actuation technologies along with an emergence of

All authors are with Toshiba Research Europe Limited, UK, Email: \{usman.raza, parag.kulkarni, mahesh.sooriyabandara\}@toshiba-trel.com. (C)2016 IEEE. Personal use of this material is permitted. Permission from IEEE must be obtained for all other uses, in any current or future media, including reprinting/republishing this material for advertising or promotional purposes, creating new collective works, for resale or redistribution to servers or lists, or reuse of any copyrighted component of this work in other works. novel communication technologies are all positive indicators, supporting the forecasted trends.

Low Power Wide Area (LPWA) networks represent a novel communication paradigm, which will complement traditional cellular and short range wireless technologies in addressing diverse requirements of IoT applications. LPWA technologies offer unique sets of features including wide-area connectivity for low power and low data rate devices, not provided by legacy wireless technologies. Their market is expected to be huge. Approximately one fourth of overall 30 billion IoT/M2M devices are to be connected to the Internet using LPWA networks using either proprietary or cellular technologies [3]. Figure 1 highlights variety of applications across several business sectors that can exploit LPWA technologies to connect their end devices. These business sectors include but not limited to smart city, personal IoT applications, smart grid, smart metering, logistics, industrial monitoring, agriculture, etc.

LPWA networks are unique because they make different tradeoffs than the traditional technologies prevalent in IoT landscape such as short-range wireless networks e.g., ZigBee, Bluetooth, Z-Wave, legacy wireless local area networks (WLANs) e.g., Wi-Fi, and cellular networks e.g. Global System for Mobile Communications (GSM), Long-Term Evolution (LTE) etc. The legacy non-cellular wireless technologies are not ideal to connect low power devices distributed over large geographical areas. The range of these technologies is limited to a few hundred meters at best. The devices, therefore, cannot be arbitrarily deployed or moved anywhere, a requirement for many applications for smart city, logistics and personal health [4]. The range of these technologies is extended using a dense deployment of devices and gateways connected using multihop mesh networking. Large deployments are thus prohibitively expensive. Legacy WLANs, on the other hand, are characterized by shorter coverage areas and higher power consumption for machine-type communication (MTC).

A wide area coverage is provided by cellular networks, a reason of a wide adoption of second generation $(2 G)$ and third generation (3G) technologies for M2M communication. However, an impending decommissioning of these technologies [5], as announced by some mobile network operators (MNOs), will broaden the technology gap in connecting low-power devices. In general, traditional cellular technologies do not achieve energy efficiency high enough to offer ten years of battery lifetime. The complexity and cost of cellular devices is high due to their ability to deal with complex waveforms, optimized for voice, high speed data services, and text. For low-power MTC, there is a clear need to strip complexity to reduce cost. Efforts in this direction are underway for cellular networks by the Third Generation Partnership Project and are covered as 

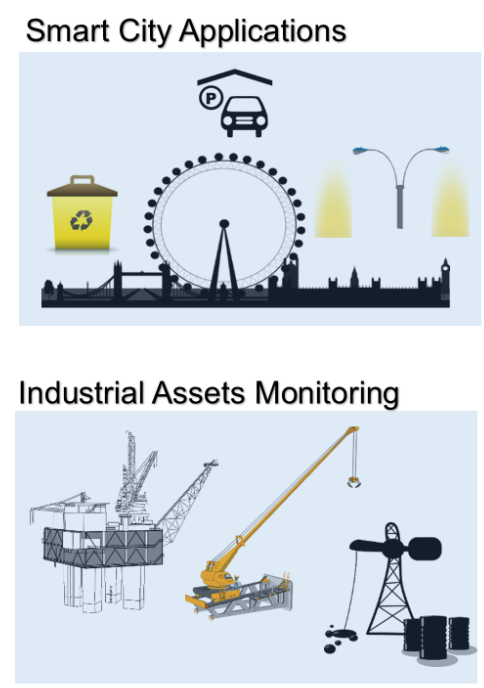

Home Automation \& Safety

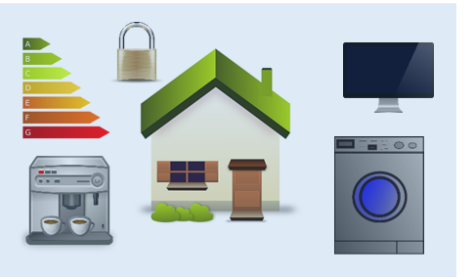

Personal loT Applications

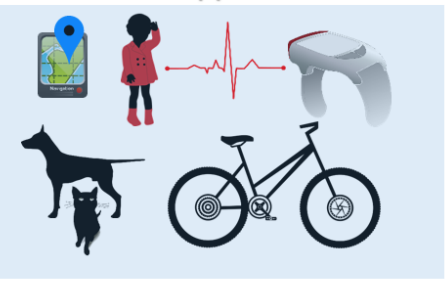

Critical Infrastructure Monitoring

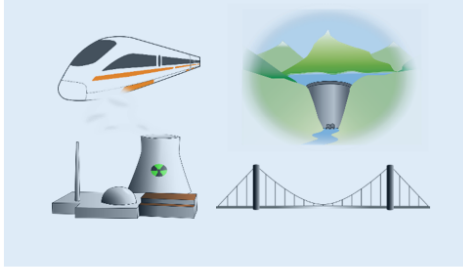

Logistics

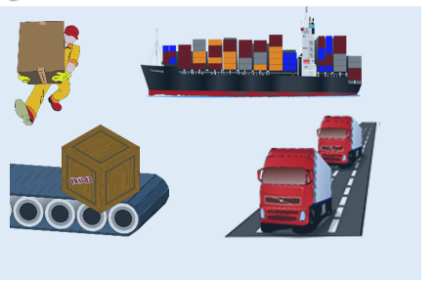

Smart Grid \& Smart Metering

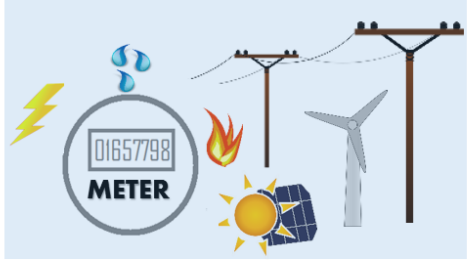

Agriculture

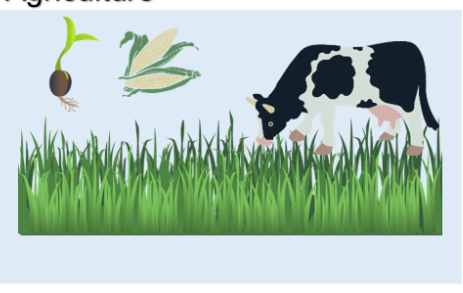

Wildlife Monitoring \& Tracking

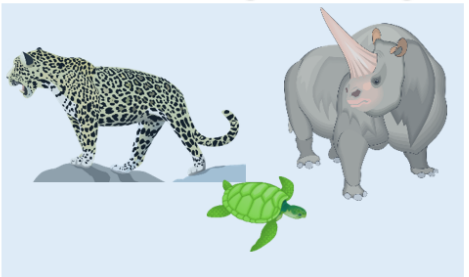

Fig. 1. Applications of LPWA technologies across different sectors

part of the discussion in Section IV.

With a phenomenal range of a few to tens of kilometers [6] and battery life of ten years and beyond, LPWA technologies are promising for the Internet of low-power, low-cost, and lowthroughput things. A very long range of LPWA technologies enables devices to spread and move over large geographical areas. IoT and M2M devices connected by LPWA technologies can be turned on anywhere and anytime to sense and interact with their environment instantly. It is worth clarifying that LPWA technologies achieve long range and low power operation at the expense of low data rate (typically in orders of tens of kilobits per seconds) and higher latency (typically in orders of seconds or minutes). Therefore it is clear that LPWA technologies are not meant to address each and every IoT use case and caters to a niche area in IoT landscape. Specifically, LPWA technologies are considered for those use cases that are delay tolerant, do not need high data rates, and typically require low power consumption and low cost, the latter being an important aspect. Such MTC application are categorized as Massive MTC [7] in contrast to Critical MTC [7] applications that require ultra-low latency and ultra high reliability. The latter are definitely out of the remit of LPWA technologies because their stringent performance requirements such as up to five nines $(99.999 \%)$ reliability and up to $1-10 \mathrm{~ms}$ latency cannot be guaranteed with a low cost and low power solution. While LPWA technologies, for this reason, are not suitable for many industrial IoT, vehicle to vehicle (V2V), and vehicle to infrastructure (V2I) applications [8], they still meet the needs of a plethora of applications for smart cities, smart metering, home automation, wearable electronics, logistics, environmental monitoring etc. (see Figure 1) that exchange small amount of data and that also infrequently. Therefore, appeal of LPWA technologies, although limited by its low data rate, is still broad. This is the reason why LPWA technologies generated so much interest after the proprietary technologies such as SIGFOX [9] and LoRa [10] hit the market.

At this moment, there are several competing LPWA technologies, each employing various techniques to achieve long range, low power operation, and high scalability. Section II presents these design goals and describes how a combination of different novel techniques actually achieves them. Section III then discusses several early proprietary LPWA technologies and their technical features, highlighting the need for standardization to flourish IoT ecosystem. To this effect, several well-known standard developing organizations (SDOs) such as European Telecommunications Standard Institute (ETSI) [11], Third Generation Partnership Project (3GPP) [12], Institute of Electrical and Electronics Engineers (IEEE) [13], and Internet Engineering Task Force (IETF) [14] are working towards the open standards for LPWA technologies. Further, multiple industrial alliances are built around individual LPWA technologies to promote new standards. LoRa $^{\mathrm{TM}}$ Alliance [15], WEIGHTLESS-SIG [16] and DASH7 Alliance [17] are a few examples of such special interest groups (SIGs). Section IV covers the standardization efforts led by all these SDOs and SIGs.

On a technical side, LPWA providers need to push innovative solutions to overcome the challenge of connecting 
massive number of IoT and M2M devices. It is indeed not an easy task especially when the heterogeneous LPWA technologies share limited radio resources to render scalable and secure connectivity to low-power and inexpensive end devices. Multiple trade-offs made by the LPWA technologies bring several challenges, which are discussed in Section $\mathrm{V}$ along with possible research directions to address them. Section VI then highlights business considerations for LPWA technologies before finally concluding this paper.

\section{Design Goals And TeChniques}

The success of LPWA technologies lies in their ability to offer low-power connectivity to massive number of devices distributed over large geographical areas at an unprecedented low-cost. This section describes the techniques LPWA technologies used to achieve these often conflicting goals. We like to highlight that LPWA technologies share some of the design goals with other wireless technologies. The key objective of LPWA technologies is, however, to achieve a long range with low power consumption and low cost unlike that of the other technologies for which achieving higher data rate, lower latency and higher reliability may be more important.

\section{A. Long range}

LPWA technologies are designed for a wide area coverage and an excellent signal propagation to hard-to-reach indoor places such as basements. Quantitatively, a $+20 \mathrm{~dB}$ gain over legacy cellular systems is targeted. This allows the end-devices to connect to the base stations at a distance ranging from a few to tens of kilometers depending on their deployment environment (rural, urban, etc.). Sub-GHz band and special modulation schemes, discussed next, are exploited to achieve this goal.

1) Use of Sub- $1 \mathrm{GHz}$ band: With an exception of a few LPWA technologies (e.g., WEIGHTLESS-W [16] and INGENU [18]), most use Sub-GHz band, which offers robust and reliable communication at low power budgets. Firstly, compared to the $2.4 \mathrm{GHz}$ band, the lower frequency signals experience less attenuation and multipath fading caused by obstacles and dense surfaces like concrete walls. Secondly, sub-GHz is less congested than $2.4 \mathrm{GHz}$, a band used by mostpopular wireless technologies e.g., Wi-Fi, cordless phones, Bluetooth, ZigBee, and other home appliances. The resulting higher reliability enables long range and low power communication. Nevertheless, the INGENU's RPMA technology [18] is an exception that still exploits $2.4 \mathrm{GHz}$ band due to more relaxed spectrum regulations on radio duty cycle and maximum transmission power in this band across multiple regions.

2) Modulation Techniques: LPWA technologies are designed to achieve a link budget of $150 \pm 10 \mathrm{~dB}$ that enables a range of a few kilometers and tens of kilometers in urban and rural areas respectively. The physical layer compromises on high data rate and slows downs the modulation rate to put more energy in each transmitted bit (or symbol). Due to this reason, the receivers can decode severely attenuated signals correctly. Typical sensitivity of state of the art LPWA receivers reaches as low as $-130 \mathrm{dBm}$. Two classes of modulation techniques namely narrowband and spread spectrum techniques have been adopted by different LPWA technologies.

Narrowband modulation techniques provide a high link budget by encoding the signal in low bandwidth (usually less than $25 \mathrm{kHz}$ ). By assigning each carrier a very narrow band, these modulation techniques share the overall spectrum very efficiently between multiple links. The noise level experienced inside a single narrowband is also minimal. Therefore, no processing gain through frequency de-spreading is required to decode the signal at the receiver, resulting in simple and inexpensive transceiver design. NB-IoT and WeIGHTLESS-P are examples of narrowband technologies.

A few LPWA technologies squeeze each carrier signal in an ultra narrow band (UNB) of width as short as $100 \mathrm{~Hz}$ (e.g., in SIGFOX), further reducing the experienced noise and increasing the number of supported end-devices per unit bandwidth. However, the effective data rate for individual end devices decreases as well, thus increasing the amount of time the radio needs to be kept ON. This low data rate in combination with spectrum regulations on sharing underlying bands may limit maximum size and transmission frequency of data packets, limiting number of business use cases. SIGFOX, WeightLESS-N and TELENSA [19] are a few examples of LPWA technologies that use UNB modulation.

Spread spectrum techniques spread a narrowband signal over a wider frequency band but with the same power density. The actual transmission is a noise-like signal that is harder to detect by an eavesdropper, more resilient to interference, and robust to jamming attacks. More processing gain is however required on the receiver side to decode the signal that is typically received below the noise floor. Spreading a narrowband signal over a wide band results in less efficient use of the spectrum. But, this problem is typically overcome by the use of multiple orthogonal sequences. As long as multiple end devices use different channels and/or orthogonal sequences, all can be decoded concurrently, resulting in a higher overall network capacity. Different variants of spread spectrum techniques are used by existing standards as discussed in Section III-B and Section III-C. Chirp Spread Spectrum (CSS) and Direct Sequence Spread Spectrum (DSSS) are used by LoRa and RPMA respectively.

\section{B. Ultra low power operation}

Ulra-low power operation is a key requirement to tap into the huge business opportunity provided by battery-powered IoT/M2M devices. A battery lifetime of 10 years or more with AA or coin cell batteries is desirable to bring the maintenance cost down.

1) Topology: While mesh topology has been extensively used to extend the coverage of short range wireless networks, their high deployment cost is a major disadvantage in connecting large number of geographically distributed devices. Further, as the traffic is forwarded over multiple hops towards a gateway, some nodes get more congested than others depending on their location or network traffic patterns. Therefore, 
they deplete their batteries quickly, limiting overall network lifetime to only a few months to years [20], [21].

On the other hand, a very long range of LPWA technologies overcomes these limitations by connecting end devices directly to base stations, obviating the need for the dense and expensive deployments of relays and gateways altogether. The resulting topology is a star that is used extensively in cellular networks and brings huge energy saving advantages. As opposed to the mesh topology, the devices need not to waste precious energy in busy-listening to other devices that want to relay their traffic through them. An always-on base station provides convenient and quick access when required by the end-devices.

In addition to star, a few LPWA technologies support tree and mesh topologies but with extra complexity in protocol design.

2) Duty Cycling: Low power operation is achieved by opportunistically turning off power hungry components of M2M/IoT devices [22], [23] e.g., data transceiver. Radio duty cycling allows LPWA end devices to turn off their transceivers, when not required. Only when the data is to be transmitted or received, the transceiver is turned on.

LPWA duty cycling mechanisms are adapted based on application, type of power source, and traffic pattern among other factors. If an application needs to transfer the data only over the uplink, the end devices may wakeup only when data is ready to be transmitted. In contrast, if downlink transmissions are required as well, the end devices make sure to listen when the base station actually transmits. The end devices achieve this by agreeing on a listening schedule. For example, the end devices may listen for a short duration after their uplink transmissions to receive a reply back. Alternatively, they may wakeup at a scheduled time agreed with the base station. For main-powered end devices requiring an ultra-low latency downlink communication, radio transceiver can stay in an always on mode. Different LPWA standards such as LORAWAN [10] define multiple classes of the end devices based on their communication needs in uplink or downlink.

In realm of LPWA technologies, duty cycling the data transceiver is not only a power saving mechanism but also a legislative requirement. Regional regulations on sharing spectrum [24] may limit the time a single transmitter can occupy to assure its coexistence with other devices sharing the same channel.

Duty cycling can also be extended beyond the transceiver to other hardware components, as explored in the context of many low-power embedded networks [25], [26]. Modular hardware design may provide ability to choose different operational modes and turn on or off individual hardware components (such as auxiliary components and storage and microcontrollers) [27]. By exploiting these power management techniques, LPWA application developers can further reduce the power consumption and increase the battery lifetime.

3) Lightweight Medium Access Control: Most-widely used Medium Access Control (MAC) protocols for cellular networks or short range wireless networks are too complex for LPWA technologies. For example, cellular networks synchronize the base stations and the user equipment (UE) accurately to benefit from complex MAC schemes that exploit frequency and time diversity. The control overhead of these schemes, while justifiable for powerful cellular UEs, is substantial for the LPWA end devices. Put differently, the control of these MAC protocols may be even more expensive than the short and infrequent machine type communication of LPWA devices. Further, a very tight synchronization needed by these schemes is difficult to be met by ultra low-cost $(\$ 1-\$ 5)$ end devices having low quality cheap oscillators. When accessing the spectrum, these devices experience drift in both time and frequency domains, making an exclusive access to the shared medium a primary challenge for the competing devices. Due to this reason, simple random access schemes are more popular for LPWA technologies.

Carrier sense multiple access with collision avoidance (CSMA/CA) is one of the most popular MAC protocols successfully deployed in WLANs and other short range wireless networks. The number of devices per base-station are limited for such networks, keeping the hidden node problem at bay. However, as the number of these devices grow in LPWA networks, carrier sensing becomes less effective and expensive [28] in reliably detecting on-going transmissions, negatively affecting the network performance. While virtual carrier sensing using Request to Send/ Clear to Send (RTS/CTS) mechanism is used to overcome this problem, it introduces extra communication overhead over the uplink and the downlink. With massive number of devices, LPWA technologies cannot usually afford this excessive signaling overhead. In addition, link asymmetry, a property of many LPWA technologies today, reduces the practicality of virtual carrier sensing. .

Due to these reasons, multiple LPWA technologies such as SIgFoX and LoRAWAN resort to the use of ALOHA, a random access MAC protocol in which end devices transmit without doing any carrier sensing. The simplicity of ALOHA is thought to keep design of transceiver simple and low cost. Nevertheless, TDMA based MAC protocols are also considered by INGENU and NB-IoT to allocate radio resources more efficiently although at the expense of more complexity and cost for end devices.

4) Offloading complexity from end devices: Most technologies simplify the design of end devices by offloading complex tasks to the base stations or to the backend system. To keep the transceiver design for end devices simple and low cost, the base stations or backend system have to be more complex. Typically, base stations exploit hardware diversity and are capable of transmitting to and listening from multiple end devices using multiple channels or orthogonal signals simultaneously. This allows end devices to send data using any available channel or orthogonal signal and still reach the base station without need for expensive signaling to initiate communication. By embedding some intelligence in backend system, end devices can further benefit from more reliable and energy efficient last mile communication. A notable example is LORAWAN in which backend system adapts communication parameters (such as data rate/ modulation parameters) to maintain good uplink and downlink connections. Furthermore, backend system is also responsible for providing support for end devices to move across multiple base stations and suppress 
duplicate receptions if any. The choice of keeping complexity at base stations and backend systems, which are fewer in number, enables low cost and low power design for many end devices.

Apart from communication, data processing can also be offloaded from end devices but we need to understand a few trade-offs first. Given the diversity of IoT applications, each may have different requirements, in particular the data reporting frequency. There may be some applications which require the end devices to report data frequently (e.g., once every few minutes). At the other extreme we may have applications that require the end devices to report data less frequently [29], [30], perhaps once a day. From an energy consumption perspective, it is a well-known fact that a communication operation consumes more energy than a processing operation. Therefore, a key question that often surfaces is whether to report all the data as it is or carry out some local processing and report the processed result (reduced need for communication). The former approach does not require any significant processing capability at the end device which implies low cost devices can be realized. However in the latter case, depending on the sophistication of processing required, the cost of the end device is likely to go up albeit reducing the energy consumption required to transport the data. The choice between the two is really driven by the underlying business case. Whilst it is always desirable to have low cost end devices especially given the large volumes of devices, it may be beneficial to have some local processing if the communication cost is substantial. Similarly, if the communication cost does not depend on the volume of data (because of flat rate pricing), then it may be beneficial to have simpler end devices. It is also necessary to estimate the costs associated with operating an end device with and without sophisticated processing. In other words, how does the cost stacks up if the end device was to be replaced often due battery depletion caused by frequent communication against deploying a slightly more expensive end device in the first place that communicates less often but does not deplete its battery that often. From a network operator's perspective, it may be desirable to reduce the amount of traffic on their network by local processing on the nodes as this may reduce the likelihood of performance issues. However, this may be undesirable if the operator's business model relies on pricing not based on volume of data.

The paradigm of processing data closer to the end device, more recently being referred to as edge computing, appears to be gaining popularity as evident from the rise of initiatives such as OpenFog [31] and Mobile Edge Computing [32]. Having said this, there is no simple one-size-fits-all binary answer to the problem of whether to transport raw data or to transport the locally processed result. As mentioned earlier, this really boils down to the requirements of the application and the analysis of return on investment (ROI) for those that want to deploy such solutions.

\section{Low Cost}

The commercial success of LPWA networks is tied to connecting a large number of end devices, while keeping the cost of hardware below $\$ 5[33]-[35]$ and the connectivity subscription per unit as low as $\$ 1$. This affordability enables LPWA technologies to not only address a wide-range of applications, but also compete favorably within the domains where the short-range wireless technologies and the cellular networks are already well-established. LPWA technologies adopt several ways to reduce the capital expenses (CAPEX) and operating expenses (OPEX) for both the end-users and network operators. The low cost design of end devices is made possible by several techniques some of which are already discussed above in II-B Use of star-type (instead of mesh) connectivity, simple MAC protocols, and techniques to offload complexity from end devices enables manufacturers to design simple and therefore low-cost end devices. Some more techniques, mechanisms and approaches are discussed as follows:

1) Reduction in hardware complexity: Compared to the cellular and the short range wireless technologies, LPWA transceivers need to process less complex waveforms. It enables them to reduce transceiver footprint, peak data rates, and memory sizes, minimizing the hardware complexity and thus the cost [1]. LPWA chip manufacturers target large number of connected end devices and can also reduce cost with economies of scale.

2) Minimum infrastructure: Traditional wireless and wired technologies suffer from limited range, requiring dense and therefore an expensive deployment of infrastructure (gateways, power lines, relay nodes etc.). However, a single LPWA base station connects tens of thousands of end devices distributed over several kilometers, significantly reducing the costs for network operators.

3) Using license-free or owned licensed bands: The cost to network operators for licensing new spectrum for LPWA technologies conflicts with low-cost deployment, short timeto-market and competitiveness of their subscription offers to customers. Therefore, most LPWA technologies considered deployment in the license-exempt bands including the industrial, scientific and medical (ISM) band or TV-white spaces. NBIoT, the LPWA standard from 3GPP, may share the cellular bands already owned by MNOs to avoid additional licensing cost. However, to get a better performance, a stand-alone licensed band can be acquired as well, a trend proprietary LPWA technologies may eventually follow to avoid performance degradation due to an increase in number of connected devices using shared spectrum.

\section{Scalability}

The support for massive number of devices sending low traffic volumes is one of the key requirements for LPWA technologies. These technologies should work well with increasing number and densities of connected devices. Several techniques are considered to cope up with this scalability problem.

1) Diversity techniques: To accommodate as many connected devices as possible, efficient exploitation of diversity in channel, time, space, and hardware is vital. Due to low-power and inexpensive nature of the end devices, much of this is achieved by cooperation from more powerful components in 
LPWA networks such as base stations and backend systems. LPWA technologies employ multi-channel and multi-antenna communication to parallelize transmissions to and from the connected devices. Further, communication is made resilient to interference by using multiple channels and doing redundant transmissions.

2) Densification: To cope up with increased density of the end devices in certain areas, LPWA networks, like traditional cellular networks, will resort to dense deployments of base stations. The problem, however, is to do so without causing too much interference between end devices and densely deployed base stations. Novel densification approaches for LPWA networks need further investigation because existing cellular techniques rely on well-coordinated radio resource management within and between cells, an assumption not true for most LPWA technologies.

3) Adaptive Channel Selection and Data Rate: Not only the LPWA systems should scale to number of connected devices, but individual links should be optimized for reliable and energy efficient communication. Adapting the modulation schemes, selecting better channels to reach distances reliably, or doing adaptive transmission power control require efficient monitoring of link qualities and coordination between end devices and network.

The extent to which adaptive channel selection and modulation is possible depends on the underlying LPWA technology. Different factors such as link asymmetry and maximum allowable radio duty cycle may limit possibility for very robust adaptive mechanisms. In the cases when the base station is unable to give feedback on quality of uplink communication and/or inform the end devices to adapt their communication parameters, the end devices resort to very simplistic mechanism to improve link quality. Such mechanism includes transmitting same packet multiple times often on multiple randomly selected channels in a hope that at least one copy reaches base station successfully. Such mechanisms arguably enhance reliability for this best-effort uplink communication, while keeping the complexity and cost of end devices very low. In the cases when some downlink communication can enable adaptation of uplink parameters, base stations or backend systems can play a vital role in selecting optimal parameters such as channel or optimal data rate to improve reliability and energy efficiency.

In summary, there is a clear trade off between network scalability and simplicity of low cost end devices. Most LPWA technologies let low-power end devices access limited radio resources in mostly uncoordinated and random fashion, limiting the number of devices that can be supported by the networks. Increasing number of recently published studies [8], [36]-[38] are revealing practical limitations on the scalability of LPWA networks. In Section V we discuss it as an interesting avenue for future research.

\section{E. Quality of Service}

LPWA technologies target diverse set of applications with varying requirements. At one extreme, it caters to delay tolerant smart metering applications, while on other end it should

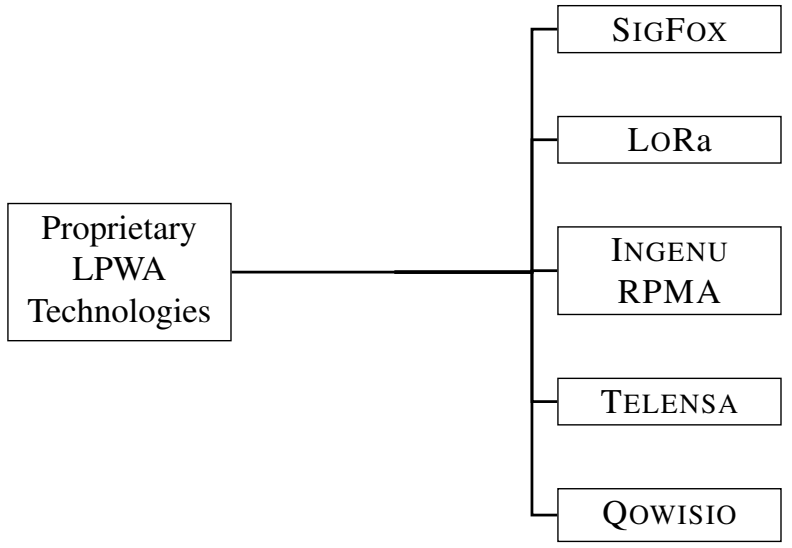

Fig. 2. Emerging proprietary LPWA technologies.

deliver the alarms generated by home security applications in minimum time. Therefore, network should provide some sort of quality of service (QoS) over the same underlying LPWA technology. For cellular standards where the underlying radio resources may be shared between LPWA and mobile broadband applications, mechanisms should be defined for co-existence of different traffic types. To the best of our knowledge, current LPWA technologies provide no or limited QoS.

\section{PROPRIETARY TECHNOLOGIES}

In this section, we highlight and compare emerging proprietary technologies shown in Figure 2 and their technical aspects summarized in Table II Some of these technologies are being made compliant to the standards proposed by the different SDOs and SIGs. We dedicate Section [V] to briefly describe these standards and their association with any proprietary technologies discussed next.

\section{A. SigFox}

SIGFOX itself or in partnership with other network operators offers an end-to-end LPWA connectivity solution based on its patented technologies. SIGFOX Network Operators (SNOs) deploy the proprietary base stations equipped with cognitive software-defined radios and connect them to the backend servers using an IP-based network. The end devices connect to these base stations using Binary Phase Shift Keying (BPSK) modulation in an ultra narrow $(100 \mathrm{~Hz}) \mathrm{SUB}-\mathrm{GHz}$ ISM band carrier. By using UNB, SIGFOX utilizes bandwidth efficiently and experiences very low noise levels, resulting in high receiver sensitivity, ultra-low power consumption, and inexpensive antenna design. All these benefits come at an expense of maximum throughput of only $100 \mathrm{bps}$. The achieved data rate clearly falls at the lower end of the throughput offered by most other LPWA technologies and thus limits the number of use-cases for SIGFOX. Further, SIGFOX initially supported only uplink communication but later evolved into a bidirectional technology, although with a significant link asymmetry. The downlink communication can only precede uplink communication after which the end device should wait to listen for a response from the base station. The number 
TABLE I

TECHNICAL SPECIFICATIONS OF VARIOUS LPWA TECHNOLOGIES (?=NOT KNOWN)

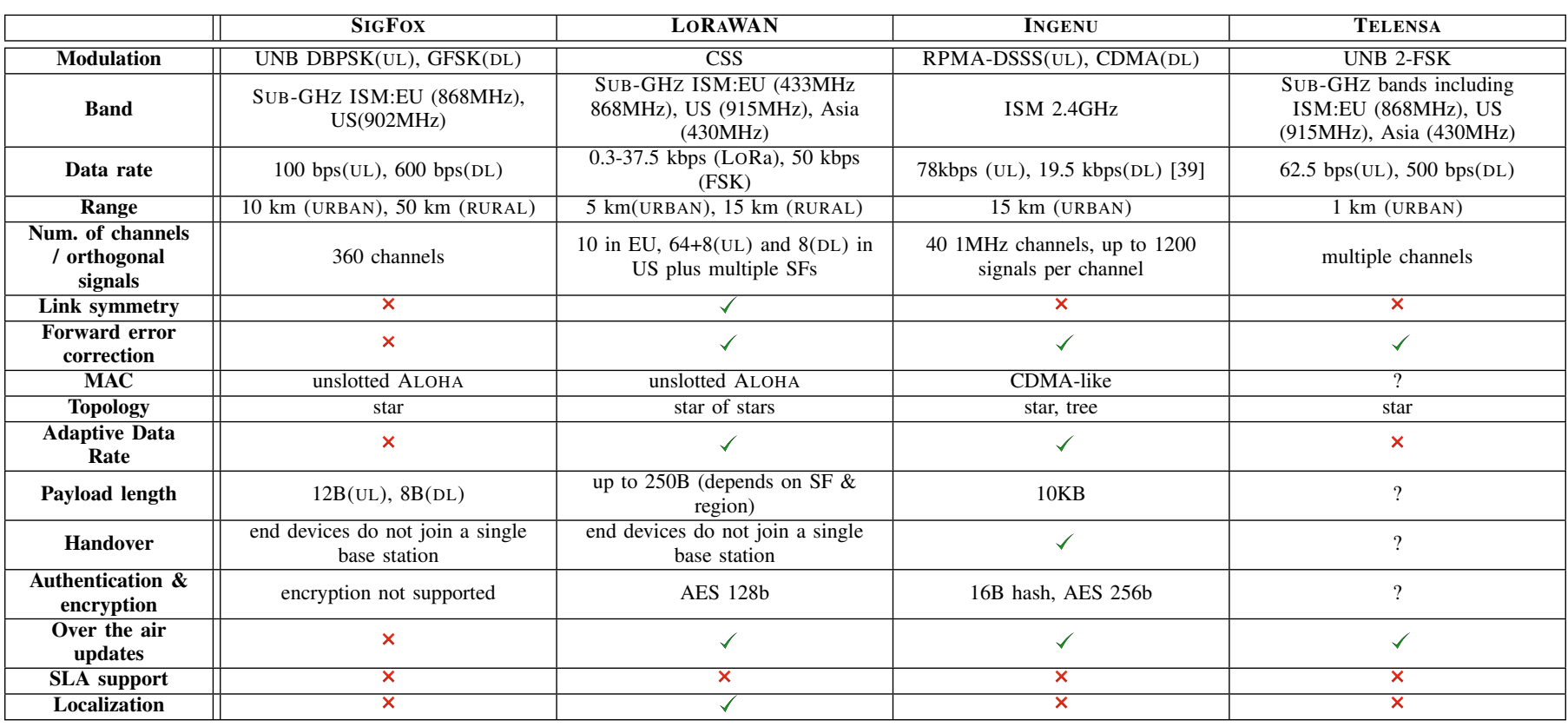

and size of messages over the uplink are limited to 14012 byte messages per day to conform to the regional regulations on use of license-free spectrum [24]. Radio access link is asymmetric, allowing transmission of maximum of only 48 bytes per day over the downlink from the base stations to the end devices. It means that acknowledging every uplink message is not supported.

Without adequate support for acknowledgments, reliability of the uplink communication is improved by using time and frequency diversity as well as redundant transmissions. A single message from an end device can be transmitted multiple times over different frequency channels. For this purpose, in Europe, the band between $868.180-868.220 \mathrm{MHz}$ is divided into $400100 \mathrm{~Hz}$ channels [40], out of which 40 channels are reserved and not used. As the base stations can scan all the channels to decode the messages, the end devices can autonomously choose a random frequency channel to transmit their messages. This simplifies the design for the end devices. Further, a single message is transmitted multiple times ( 3 by default) to increase the probability of successful reception by the base stations.

\section{B. $\operatorname{LOR} a$}

LoRa is a physical layer technology that modulates the signals in SUB-GHz ISM band using a proprietary spread spectrum technique [41] developed and commercialized by Semtech Corporation [42]. A bidirectional communication is provided by a special chirp spread spectrum (CSS) technique, which spreads a narrow band input signal over a wider channel bandwidth. The resulting signal has noise like properties, making it harder to detect or jam. The processing gain enables resilience to interference [43] and noise.

The transmitter makes the chirp signals vary their frequency over time without changing their phase between adjacent symbols. As long as this frequency change is slow enough so to put higher energy per chirp symbol, distant receivers can decode a severely attenuated signal several $\mathrm{dBs}$ below the noise floor. LoRa supports multiple spreading factors (between 7-12) to decide the tradeoff between range and data rate. Higher spreading factors delivers long range at an expense of lower data rates and vice versa. LORa also combines Forward Error Correction (FEC) with the spread spectrum technique to further increase the receiver sensitivity. The data rate ranges from $300 \mathrm{bps}$ to $37.5 \mathrm{kbps}$ depending on spreading factor and channel bandwidth. Further, multiple transmissions using different spreading factors can be received simultaneously by a LoRa base station. In essence, multiple spreading factors provide a third degree of diversity after time and frequency.

A few studies evaluated LORAWAN in real world environments including outdoor [6], [44], [45] and even indoor [46] settings. The work in [44] evaluates LoRa and SIGFOX through experiments carried out from a test deployment in Ireland. Findings indicate that a LoRa base station deployed at $470 \mathrm{~m}$ above sea level could serve a coverage area of 1380 square kilometers in the test setup and that SIGFOX technology was able to provide a $25 \mathrm{~km}$ test link between a client using $14 \mathrm{dBm}$ and the base station with an signal to noise ratio consistently exceeding $20 \mathrm{~dB}$ being measured in the tests performed. Another study in [6] observed $15 \mathrm{~km}$ and $30 \mathrm{~km}$ communication ranges for LORAWAN on ground and water respectively in Oulu Finland. Furthermore, in another study [47] conducted at a university, end devices transmitted at $14 \mathrm{dBm}$ using highest spreading factor (12) to the base station that was located within $420 \mathrm{~m}$ radius. The packet delivery ratio at the base station is recorded to be $96.7 \%$.

The messages transmitted by the end devices are received by not a single but all the base stations in the range, giving rise to "star-of-stars" topology. By exploiting reception diversity this way, LoRa improves ratio of successfully received messages. However, achieving this requires multiple base stations in 
the neighborhood that may increase CAPEX and OPEX. The resulting duplicate receptions are filtered out in the backend system. Further, LoRa exploits these multiple receptions of same message at different base stations for localization of the transmitting end device. For this purpose, a time difference of arrival (TDOA) based localization technique supported by very accurate time synchronization between multiple base station is used.

A special interest group constituted by several commercial and industrial partners dubbed as $\mathrm{LORa}^{\mathrm{TM}}$ Alliance proposed LORAWAN, an open standard defining architecture and layers above the LoRa physical layer. We briefly describe LORAWAN under standards in Section IV

\section{INGENU RPMA}

INGENU (formerly known as On-Ramp Wireless) proposed a proprietary LPWA technology, which unlike most other technologies does not rely on better propagation properties of SuB-GHz band. Instead it operates in $2.4 \mathrm{GHz}$ ISM band and leverages more relaxed regulations on the spectrum use across different regions [18], [24]. To offer an example, the regulations in USA and Europe do not impose a maximum limit on duty cycle for $2.4 \mathrm{GHz}$ band, enabling higher throughput and more capacity than other technologies operating in SUB-GHz band.

Most importantly, INGENU uses a patented physical access scheme named as Random Phase Multiple Access (RPMA) [48] Direct Sequence Spread Spectrum, which it employs for uplink communication only. As a variation of Code Division Multiple Access (CDMA) itself, RPMA enables multiple transmitters to share a single time slot. However, RPMA first increases time slot duration of traditional CDMA and then scatters the channel access within this slot by adding a random offset delay for each transmitter. By not granting channel access to the transmitters exactly at once (i.e., at the beginning of a slot), RPMA reduces overlapping between transmitted signals and thus increases signal to interference ratio for each individual link [18]. On the receiving side, the base stations employ multiple demodulators to decode signals arriving at different times within a slot. INGENU provides bidirectional communication, although with a slight link asymmetry. For downlink communication, base stations spreads the signals for individual end devices and then broadcast them using CDMA.

RPMA is reported to achieve up to $-142 \mathrm{dBm}$ receiver sensitivity and $168 \mathrm{~dB}$ link budget [18]. Further, the end devices can adjust their transmit power for reaching closest base station and limiting interference to nearby devices.

INGENU leads efforts to standardize the physical layer specifications under IEEE 802.15.4k standard. RPMA technology is made compliant to the IEEE $802.15 .4 \mathrm{k}$ specifications.

\section{Telensa}

TELENSA [49] provides end-to-end solutions for LPWA applications incorporating fully designed vertical network stacks with a support for integration with third party software.

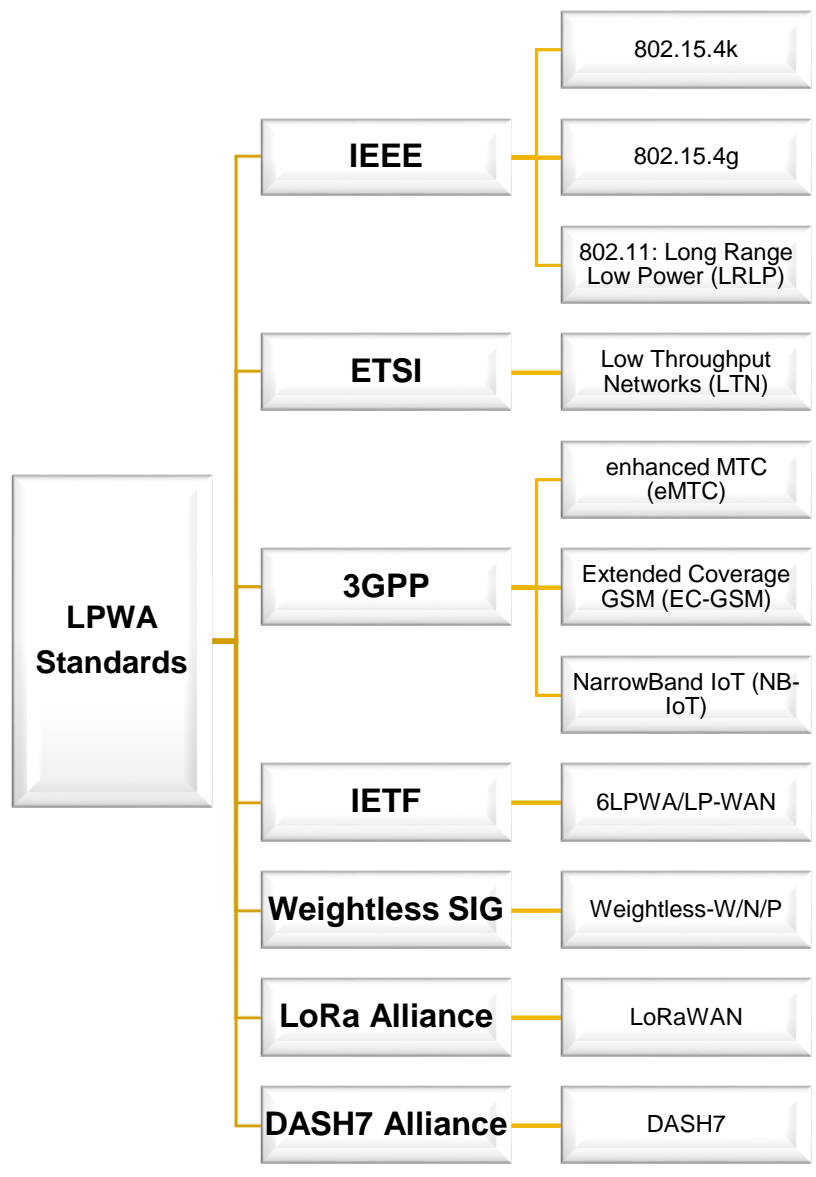

Fig. 3. LPWA standards and their developing organizations

For a wireless connectivity between their end devices and the base stations, TELENSA designed a proprietary UNB modulation technique [19], which operates in license-free SUBGHz ISM band at low data rates. While less is known about the implementation of their wireless technology, TELENSA aims to standardize its technology using ETSI Low Throughput Networks (LTN) specifications for an easy integration within applications.

TELENSA currently focuses on a few smart city applications such as intelligent lighting, smart parking, etc. To strengthen their LPWA offerings in intelligent lighting business, TELENSA is involved with TALQ consortium [50] in defining standards for monitoring and controlling outdoor lighting systems.

\section{E. QOWISIO}

QOWISIO deploys dual-mode LPWA networks combining their own proprietary UNB technology with LoRa. It provides LPWA connectivity as a service to the end users: It offers end devices, deploys network infrastructure, develops custom applications, and hosts them at a backend cloud. Less is however known about the technical specifications of their underlying UNB technology and other system components. 


\section{STANDARDS}

A plethora of standardization efforts are undertaken by different established standardization bodies including Institute of Electrical and Electronics Engineers (IEEE), European Telecommunications Standard Institute (ETSI), and The Third Generation Partnership Project (3GPP) along with industrial consortia such as WEIGHTLESS-SIG, LORa ${ }^{\mathrm{TM}}$ Alliance, and DASH7 Alliance. Figure 3 organizes the proposed standards according to their developing organizations, while Table II summarizes technical specifications of different standards. A qualitative comparison of some LPWA technologies can be found in [51]. Most of these efforts also involve several proprietary LPWA connectivity providers discussed in the previous section. The objectives of these SDOs and SIGs are quite diverse. In the long run, it is hoped that adoption of these standards will likely reduce the fragmentation of LPWA market and enable co-existence of multiple competing technologies.

\section{A. IEEE}

IEEE is extending range and reducing power consumption of their 802.15.4 [52] and 802.11 [53] standards with the set of new specifications for the physical and the MAC layers. Two LPWA standards are proposed as amendments to IEEE 802.15.4 base standard for Low-Rate Wireless Personal Area Networks (LR-WPANs), which we will cover in this section. Along with this, the efforts on amending IEEE 802.11 standard for wireless local area networks (WLANs) for longer range are also briefly described.

1) IEEE 802.15.4k: Low Energy, Critical Infrastructure Monitoring Networks.: IEEE 802.15.4k Task Group (TG4k) proposes a standard for low-energy critical infrastructure monitoring (LECIM) applications to operate in the ISM bands (SUB-GHz and $2.4 \mathrm{GHz}$ ). This was a response to the fact that the earlier standard falls short on range and the node densities required for LPWA applications. IEEE 802.15.4k amendment bridges this gap by adopting DSSS and FSK as two new PHY layers. Multiple discrete channel bandwidths ranging from $100 \mathrm{kHz}$ to $1 \mathrm{MHz}$ can be used. The MAC layer specifications are also amended to address the new physical layers. The standard supports conventional CSMA/CA without priority channel access (PCA), CSMA, and ALOHA with PCA. With PCA, the devices and base stations can prioritize their traffic in accessing the medium, providing a notion of quality of service. Like most LPWA standards, end-devices are connected to the base stations in a star topology and are capable of exchanging asynchronous and scheduled messages.

An IEEE 802.15.4k based LPWA deployment for air quality monitoring is elaborated in [54]. A star topology network was deployed wherein 1 access point and 5 nodes were deployed within a $3 \mathrm{~km}$ radius area from the center of the university campus. The access point operates in the $433 \mathrm{MHz}$ spectrum. Using a transmit power of $15 \mathrm{dBm}$ the transceiver can support different sensitivities depending on the data rate requirements, e.g. sensitivities of $-129 \mathrm{dBm},-123 \mathrm{dBm}$ and $-110 \mathrm{dBm}$ can be achieved for data rates corresponding to $300 \mathrm{bps}, 1.2 \mathrm{kbps}$ and $50 \mathrm{kbps}$ respectively.
INGENU, the provider of the RPMA LPWA technology [18], is a proponent of this standard. The PHY and MAC layers of INGENU's LPWA technology are compliant with this standard.

2) IEEE 802.15.4g: Low-Data-Rate, Wireless, Smart Metering Utility Networks: IEEE 802.15 WPAN task group 4g (TG4g) proposes first set of PHY amendments to extend the short range portfolio of IEEE 802.15.4 base standard. The release of standard in April 2012 [55] addresses the process-control applications such as smart metering networks, which are inherently comprised of massive number of fixed end devices deployed across cities or countries. The standard defines three PHY layers namely FSK, Orthogonal FrequencyDivision Multiple Access (OFDMA), and offset Quaternary Phase Shift Keying (QPSK), which support multiple data rates ranging from $40 \mathrm{kbps}$ to $1 \mathrm{Mbps}$ across different regions. With an exception of a single licensed band in USA, the PHY predominantly operates in ISM (SUB-GHz and $2.4 \mathrm{GHz}$ ) bands and thus co-exists with other interfering technologies in the same band. The PHY is designed to deliver frames of size up to 1500 bytes so to avoid fragmenting Internet Protocol (IP) packets.

The changes in the MAC layer to support the new PHYs are defined by IEEE $802.15 .4 \mathrm{e}$ and not by IEEE $802.15 .4 \mathrm{~g}$ standard itself.

3) IEEE 802.11: Wireless Local Area Networks: WLAN technologies will play an important role in IoT [56]. The efforts for extending range and decreasing power consumption for WLANs are made by the IEEE 802.11 Task Group AH (TGah) and the IEEE 802.11 Topic Interest Group (TIG) in Long Range Low Power (LRLP).

TGah [57] proposed the IEEE 802.11ah specifications for PHY and MAC to operate long range Wi-Fi operation in SUB-GHz ISM band. Compared to IEEE 802.11ac standard, several new features were introduced to achieve $1 \mathrm{~km}$ range in outdoor environments and the data rate in excess of 100 kbps. The PHY adopts OFDM that transmit at the rate 10 times slower than IEEE 802.11ac, an earlier standard, so to extend the communication range. At the MAC layer, overheads associated with frames, headers and beacons are reduced to prolong battery powered operation [58]. MAC protocol is tailored to thousands (8191) of connected end devices so that it reduces the resulting collisions among them. End devices are enabled with mechanisms to save energy during the inactive periods but yet retain their connection/synchronization with the access points. With all these new power saving and range enhancements, IEEE 802.11ah indeed provides significantly longer range and lower energy consumption than other WLAN standards, ZigBee, and Bluetooth but not as much as the other LPWA technologies discussed in this paper. Due to this reason, increasing number of recently published studies [59], [60] and IETF draft documents [61], [62] do not enlist IEEE 802.11ah as a LPWA technology. In fact, IEEE 802.11ah caters to those applications that require relatively higher bandwidth at the expense of higher power consumption than the other LPWA technologies.

Feasibility of using IEEE 802.11 ah for IoT/M2M use cases is studied in [63]. The authors show that when using the 900 
TABLE II

TECHNICAL SPECIFICATIONS OF VARIOUS LPWA STANDARDS

\begin{tabular}{|c|c|c|c|c|c|c|}
\hline \multirow{2}{*}{ Standard } & \multicolumn{2}{|c|}{ IEEE } & \multicolumn{3}{|c|}{ WEIGHTLESS-SIG } & \multirow{2}{*}{$\begin{array}{l}\text { DASH7 Alliance } \\
\text { DASH7 }\end{array}$} \\
\hline & $802.15 .4 k$ & $802.15 .4 \mathrm{~g}$ & WEIGHTLESS-W & WEIGHTLESS-N & WEIGHTLESS-P & \\
\hline Modulation & DSSS, FSK & $\begin{array}{l}\text { MR-(FSK, OFDMA, } \\
\text { OQPSK) }\end{array}$ & $\begin{array}{l}\text { 16-QAM, BPSK, } \\
\text { QPSK, DBPSK }\end{array}$ & UNB DBPSK & GMSK, offset-QPSK & GFSK \\
\hline Band & $\begin{array}{l}\text { ISM SUB-GHz \& } \\
2.4 \mathrm{GHz}\end{array}$ & $\begin{array}{l}\text { ISM SUB-GHz \& } \\
2.4 \mathrm{GHz}\end{array}$ & $\begin{array}{l}\text { TV white spaces } \\
\text { 470-790MHz }\end{array}$ & $\begin{array}{c}\text { ISM SUB-GHz EU } \\
\text { (868MHz), US } \\
\text { (915MHz) }\end{array}$ & $\begin{array}{l}\text { SUB-GHZ ISM or } \\
\text { licensed }\end{array}$ & $\begin{array}{l}\text { SuB-GHz 433MHz, } \\
868 \mathrm{MHz}, 915 \mathrm{MHz}\end{array}$ \\
\hline Data rate & 1.5 bps- $128 \mathrm{kbps}$ & 4.8 kbps- 800 kbps & 1 kbps-10 Mbps & 30 kbps-100 kbps & 200 bps-100kbps & $9.6,55.6,166.7 \mathrm{kbps}$ \\
\hline Range & $5 \mathrm{~km}$ (URBAN) & up to several $\mathrm{kms}$ & $5 \mathrm{~km}$ (URBAN) & $3 \mathrm{~km}$ (URBAN) & $2 \mathrm{~km}$ (URBAN) & $0-5 \mathrm{~km}$ (URBAN) \\
\hline $\begin{array}{c}\text { Num. of } \\
\text { channels / } \\
\text { orthogonal } \\
\text { signals }\end{array}$ & \multicolumn{2}{|c|}{$\begin{array}{l}\text { multiple channels. } \\
\text { Number depends on channel \& modulation }\end{array}$} & $\begin{array}{c}16 \text { or } 24 \\
\text { channels(UL) }\end{array}$ & $\begin{array}{l}\text { multiple } 200 \mathrm{~Hz} \\
\text { channels }\end{array}$ & $\begin{array}{l}\text { multiple } 12.5 \mathrm{kHz} \\
\text { channels }\end{array}$ & $\begin{array}{c}3 \text { different channel } \\
\text { types (number } \\
\text { depends on type \& } \\
\text { region) }\end{array}$ \\
\hline $\begin{array}{c}\text { Forward error } \\
\text { correction }\end{array}$ & $\checkmark$ & $\checkmark$ & $\checkmark$ & $x$ & $\checkmark$ & $\checkmark$ \\
\hline MAC & $\begin{array}{c}\text { CSMA/CA, } \\
\text { CSMA/CA or } \\
\text { ALOHA with PCA }\end{array}$ & CSMA/CA & TDMA/FDMA & slotted ALOHA & TDMA/FDMA & CSMA/CA \\
\hline Topology & star & $\begin{array}{c}\text { star, mesh, } \\
\text { peer-to-peer (depends } \\
\text { on upper layers) }\end{array}$ & star & star & star & tree, star \\
\hline Payload length & $2047 \mathrm{~B}$ & $2047 \mathrm{~B}$ & $>10 \mathrm{~B}$ & $20 \mathrm{~B}$ & $>10 \mathrm{~B}$ & $256 \mathrm{~B}$ \\
\hline $\begin{array}{l}\text { Authentication } \\
\text { \& encryption }\end{array}$ & AES $128 b$ & AES $128 b$ & AES $128 b$ & AES $128 b$ & AES 128/256b & AES 128b \\
\hline
\end{tabular}

$\mathrm{MHz}$ band, for the downlink case, it is straightforward to achieve a $1 \mathrm{~km}$ range and higher than $100 \mathrm{kbps}$ data rate as the AP uses higher transmit power $(20-30 \mathrm{dBm})$. However, for the uplink case, it is quite challenging to achieve these targets as the clients operate a low power $(0 \mathrm{dBm})$ and are to be duty cycled to enable years of battery operation. In such a case, range of up to $400 \mathrm{~m}$ was achieved with the authors highlighting that use of coding schemes, higher transmit power and higher gain antennas could potentially help to improve this. However this may come at the cost of reduced battery life at the clients which may not be desirable. They also suggest that if the reliability requirements are reduced, range can be further increased, e.g. they were able to achieve $1 \mathrm{~km}$ range for a link reliability less than $60 \%$.

A new Topic Interest Group (TIG) was setup under the remit of 802.11 in 2016 to explore feasibility of a new standard for Long Range Low Power (LRLP) [64]. At an early stage of this work, the TIG had defined some use cases and functional requirements for this technology in [65] but could not clearly justify need for this activity within the IEEE LAN/MAN Standards Committee (LMSC). Therefore, the work on LRLP came to a premature end.

\section{B. ETSI}

ETSI leads efforts to standardize a bidirectional low data rate LPWA standard. The resulting standard dubbed as Low Throughput Network (LTN) was released in 2014 in the form of three group specifications. These specifications define i) the use cases [66] ii) the functional architecture [67], and iii) the protocols and interfaces [68]. One of its primary objectives is to reduce the electromagnetic radiation by exploiting short payload sizes and low data rates of M2M/IoT communication.

Apart from the recommendation on the air interfaces, LTN defines various interfaces and protocols for the cooperation between end-devices, base stations, network server, and operational and business management systems.

Motivated by the fact that the emerging LPWA networks use both ultra narrow band (e.g.,SigFox, TELENSA) and orthogonal sequence spread spectrum (OSSS) (e.g., LoRa) modulation techniques, LTN standard does not restrict itself to a single category. It provides flexibility to LPWA operators to design and deploy their own proprietary UNB or OSSS modulation schemes in SUB-GHz ISM band as long as the end-devices, base stations and the network servers implement the interfaces described by the LTN specifications [66]-[68]. These specifications recommend using BPSK in uplink and GFSK in downlink for a UNB implementation. Alternatively, any OSSS modulation scheme can be used to support bidirectional communication. Data encryption as well as user authentication procedures are defined as a part of the LTN specifications.

Several providers of LPWA technologies such as SIGFox, TELENSA, and Semtech are actively involved with ETSI for standardization of their technologies.

\section{C. $3 G P P$}

To address M2M and IoT market, 3GPP is evolving its existing cellular standards to strip complexity and cost, improve the range and signal penetration, and prolong the battery lifetime. Its multiple licensed solutions such as Long Term Evolution (LTE) enhancements for Machine Type Communications (eMTC), Extended Coverage GSM (EC-GSM), and NarrowBand IoT (NB-IoT) offer different trade-offs between cost, coverage, data rate, and power consumption to address diverse needs of IoT and M2M applications. However, a common goal of all these standards is to maximize the re-use of the existing cellular infrastructure and owned radio spectrum.

1) LTE enhancements for Machine Type Communications (eMTC): Conventional LTE end devices offer high data rate services at a cost and power consumption not acceptable for several MTC use cases. To reduce the cost while being compliant to LTE system requirements, 3GPP reduces the peak data rate from LTE Category 1 to LTE Category 0 and then to LTE Category M, the different stages in the LTE evolution process. Further cost reduction is achieved by supporting optional half duplex operation in Category 0 . This 
choice reduces the complexity of modem and antenna design. From Category 0 to Category M1 (also known as eMTC), a more pronounced drop in the receive bandwidth from $20 \mathrm{MHz}$ to $1.4 \mathrm{MHz}$ in combination with a reduced transmission power will result in more cost-efficient and low-power design.

To extend the battery lifetime for eMTC, 3GPP adopts two features namely Power Saving Mode (PSM) and extended Discontinuous Reception (eDRx). They enable end devices to enter in a deep sleep mode for hours or even days without losing their network registration. The end devices avoid monitoring downlink control channel for prolonged periods of time to save energy. The same power saving features are exploited in EC-GSM described next.

2) EC-GSM: While Global System for Mobile Communications (GSM) is announced to be decommissioned in certain regions, Mobile Network Operators (MNOs) may like to prolong their operation in few markets. With this assumption, 3GPP is in process of proposing the extended coverage GSM (EC-GSM) standard that aims to extend the GSM coverage by $+20 \mathrm{~dB}$ using SuB-GHz band for better signal penetration in indoor environments. A link budget in the range of 154 $\mathrm{dB}-164 \mathrm{~dB}$ is aimed depending on the transmission power. With only a software upgrade of GSM networks, the legacy GPRS spectrum can pack the new logical channels defined to accommodate EC-GSM devices. EC-GSM exploits repetitive transmissions and signal processing techniques to improve coverage and capacity of legacy GPRS. Two modulation techniques namely Gaussian Minimum Shift Keying (GMSK) and 8-ary Phase Shift Keying (8PSK) provide variable data rates with the peak rate of $240 \mathrm{kbps}$ with the latter technique. The standard was released in mid 2016 and aims to support 50k devices per base station and enhanced security and privacy features compared to conventional GSM based solutions.

3) NB-IoT: NB-IoT is a narrow-band technology that was made available as a part of Release-13 around mid 2016. NBIoT aims at enabling deployment flexibility, long battery life, low device cost and complexity and signal coverage extension. NB-IoT is not compatible with $3 \mathrm{G}$ but can coexist with GSM, GPRS and LTE. NB-IoT can be supported with only a software upgrade on top of existing LTE infrastructure. It can be deployed inside a single GSM carrier of $200 \mathrm{kHz}$, inside a single LTE physical resource block (PRB) of $180 \mathrm{kHz}$ or inside an LTE guard band. Compared to eMTC, NB-IoT cuts the cost and energy consumption further by reducing the data rate and bandwidth requirements (needs only $180 \mathrm{kHz}$ ) and simplifying the protocol design and mobility support. Further, a standalone deployment in a dedicated licensed spectrum is supported.

NB-IoT aims for a $164 \mathrm{~dB}$ coverage, serving up to 50k end devices per cell with the potential for scaling up the capacity by adding more NB-IoT carriers. NB-IoT uses single-carrier Frequency Division Multiple Access (FDMA) in uplink and Orthogonal FDMA (OFDMA) in downlink [69]. The data rate is limited to $250 \mathrm{kbps}$ for the multi-tone downlink communication and to $20 \mathrm{kbps}$ for the single-tone uplink communication. As highlighted in [70], for a $164 \mathrm{~dB}$ coupling loss, an NBIo $T$ based radio can achieve a battery life of 10 years when transmitting 200 bytes of data per day on average. For an in-depth look into NB-IoT, we refer the interested reader to [70]. Further [69] compares the different cellular based LPWA options covered in this section.

Further to publication of Release-13 specifications, NBIoT standard has been critiqued in [71]). We summarize this critique as follows:

- Only half the messages are acknowledged in NB-IoT due to limited downlink capacity. This implies the inability to realize IoT applications that require acknowledging of all uplink data traffic unless the application implements some form of reliability mechanisms. The latter could result in increased application complexity and higher energy consumption due to extra processing.

- Use of packet aggregation (combining multiple packets and sending them as a single larger packet) in 3GPP based solutions improves efficiency but comes at the cost of extra latency that may be undesirable for delay sensitive IoT applications.

- NB-IoT traffic is best effort and therefore during times of heavy voice/data traffic, dynamically reallocating spectrum to relieve congestion for the latter class of traffic may impact NB-IoT application performance. Further, once deployed an NB-IoT device is likely to stay put for 10-20 years, an order of magnitude higher device upgrade cycle when compared to traditional mobile phones (typically 2 years). Some applications may take longer to break-even and provide a return on investment. Moreover, if new cellular generations come along, there could be questions with respect to the longevity of the deployed solution, e.g. a situation similar to some operators phasing out their GSM networks to reclaim the spectrum for LTE. This could leave the customers stranded since it may not be trivial/economically feasible to upgrade the end points, a valid argument.

- The lack of commercial deployments leaves open questions on the actual battery life and performance attainable in real world conditions.

\section{IETF}

IETF aims to support LPWA ecosystem of dominantly proprietary technologies by standardizing end-to-end IP-based connectivity for ultra-low power devices and applications. IETF has already designed the IPv6 stack for Low power Wireless Personal Area Networks (6LoWPAN). However, these standardization efforts focus on legacy IEEE 802.15 .4 based wireless networks, which support relatively higher data rates, longer payload sizes and shorter ranges than most LPWA technologies today. However, distinct features of LPWA technologies pose real technical challenges for the IP connectivity. Firstly, LPWA technologies are heterogeneous: every technology manipulates data in different formats using different physical and MAC layers. Secondly, most technologies use the ISM bands, which are subject to strict regional regulations, limiting maximum data rate, time-on-air, and frequency of data transmissions. Third, many technologies are characterized by a strong link asymmetry between uplink and downlink, usually limiting downlink capabilities. Thus, the proposed 
IP stacks should be lightweight enough to confine within these very strict limitations of the underlying technologies. Unfortunately, these challenges are not yet addressed in earlier IETF standardization efforts.

A working group on Low-Power Wide Area Networks (LPWAN) [62] under IETF umbrella was formed in April 2016. This group identified challenges and the design space for IPv6 connectivity for LPWA technologies in [61]. Future efforts may likely culminate into multiple standards defining a full IPv6 stack for LPWA (6LPWA) that can connect LPWA devices with each other and their external ecosystem in a secure and a scalable manner. More specific technical problems to be addressed by this IETF group are described as follows:

- Header compression. The maximum payload size for LPWA technologies is limited. The header compression techniques should be tailored to these small payload sizes as well as sparse and infrequent traffic of LPWA devices.

- Fragmentation and reassembly. Most LPWA technologies do not natively support fragmentation and reassembly at Layer 2 (L2). Because IPv6 packets are often too big to fit in a single L2 packet, the mechanisms for fragmentation and reassembly of IPv6 packets are to be defined.

- Management. To manage end devices, applications, base stations, and servers, there is a need for ultra-lightweight signaling protocols, which can operate efficiently over the constrained L2 technology. To this effect, IETF may look into efficient application-level signaling protocols [72].

- Security, integrity, and privacy. The IP connectivity should preserve security, integrity, and privacy of data exchanged over LPWA radio access networks and beyond. Most LPWA technologies use symmetric key cryptography, in which end devices and the networks share the same secret key. More robust and resilient techniques and mechanisms may be investigated.

\section{E. LoR $a^{\mathrm{TM}}$ Alliance}

As described in Section III. LoRa is a proprietary physical layer for LPWA connectivity. However, the upper layers and the system architecture are defined by LoRa ${ }^{\mathrm{TM}}$ Alliance under

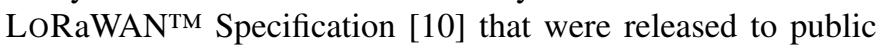
in July 2015.

A simple AlOHA scheme is used at the MAC layer that in combination with LoRa physical layer enables multiple devices to communicate at the same time but using different channels and/or orthogonal codes (i.e., spreading factors). End devices can hop on to any base station without extra signaling overhead. The base stations connect end devices via a backhaul to network server, the brain of the LoRAWAN system that suppresses duplicate receptions, adapts radio access links, and forwards data to suitable application servers. Application servers then process the received data and perform user defined tasks.

LORAWAN anticipates that the devices will have different capabilities as per application requirements. Therefore,
LORAWAN defines three different classes of end-devices, all of which support bidirectional communication but with different downlink latency and power requirements. Class A device achieves the longest lifetime but with the highest latency.It listens for a downlink communication only shortly after its uplink transmission. Class B device, in addition, can schedule downlink receptions from base station at certain time intervals. Thus, only at these agreed-on epochs, applications can send control messages to the end devices (for possibly performing an actuation function). Lastly, Class C device is typically mains-powered, having capability to continuously listen and receive downlink transmissions with the shortest possible latency at any time.

LORAWAN standard uses symmetric-key cryptography to authenticate end devices with the network and preserve the privacy of application data.

\section{F. WeightLESS-SIG}

WeIGHTLESS Special Interest Group [16] proposed three open LPWA standards, each providing different features, range and power consumption. These standards can operate in license-free as well as in licensed spectrum.

WeightLeSS-W leverages excellent signal propagation properties of TV white-spaces. It supports several modulation schemes including 16-Quadrature Amplitude Modulation (16QAM) and Differential-BPSK (DBPSK) and a wide range of spreading factors. Depending on the link budget, the packets having sizes in upwards of 10 bytes can be transmitted at a rate between $1 \mathrm{kbps}$ and $10 \mathrm{Mbps}$. The end devices transmit to base stations in a narrow band but at a lower power level than the base stations to save energy. WEIGHTLESS-W has a one drawback. The shared access of the TV white spaces is permitted only in few regions, therefore WEIGHTLESS-SIG defines the other two standards in ISM band, which is globally available for shared access.

WeIGHTLESS-N is a UNB standard for only one-way communication from end devices to a base station, achieving significant energy efficiency and lower cost than the other WEIGHTLESS standards. It uses DBPSK modulation scheme in SUB-GHz bands. One-way communication, however, limits the number of use cases for WEIGHTLESS-N.

WeightLeSS-P blends two-way connectivity with two nonproprietary physical layers. It modulates the signals using GMSK and Quadrature Phase Shift Keying (QPSK), two well known schemes adopted in different commercial products. Therefore, the end devices do not require a proprietary chipset. Each single $12.5 \mathrm{kHz}$ narrow channel in SuB-GHz ISM band offers a data rate in the range between $0.2 \mathrm{kbps}$ to 100 kbps. A full support for acknowledgments and bidirectional communication capabilities enable over-the-air upgrades of firmware.

Like LORAWAN, all WEIGHTLESS standards employ symmetric key cryptography for authentication of end devices and integrity of application data. 


\section{G. DASH7 Alliance}

The DASH7 Alliance is an industry consortium that defines a full vertical network stack for LPWA connectivity known as DASH7 Alliance Protocol (D7AP) [73]. With its origin in the ISO/IEC 18000-7 standard [74] for the air interface for active radio frequency identification (RFID) devices, D7AP has evolved into a stack that provides mid-range connectivity to low-power sensors and actuators [73].

DASH7 employs narrow band modulation scheme using two-level GFSK in SUB-GHz bands. Compared to most other LPWA technologies, DASH7 has a few notable differences. First it uses a tree topology by default with an option to choose star layout as well. In the former case, the end devices are first connected to duty-cycling sub-controllers, which then connect to the always ON base stations. This duty cycling mechanism brings more complexity to the design of the upper layers. Second, DASH7 MAC protocol forces the end devices to check the channel periodically for possible downlink transmissions, adding significant idle listening cost. By doing so, DASH7 gets much lower latency for downlink communication than other LPWA technologies but at an expense of higher energy consumption. Third, unlike other LPWA technologies, DASH7 defines a complete network stack, enabling applications and end devices to communicate with each other without having to deal with intricacies of the underlying physical or MAC layers.

DASH7 implements support for forward error correction and symmetric key cryptography.

\section{Challenges and Open Research Directions}

LPWA players are striving hard to innovate solutions that can deliver the so-called carrier grade performance. To this effect, device manufacturers, network operators, and system integration experts have concentrated their efforts on cheap hardware design, reliable connectivity, and full end-to-end application integration. On the business side, the proprietary solution providers are in a rush to bring their services to the market and capture their share across multiple verticals. In this race, it is easy but counter-productive to overlook important challenges faced by LPWA technologies. In this section, we highlight these challenges and some research directions to overcome them and improve performance in long-term.

\section{A. Scaling networks to massive number of devices}

LPWA technologies will connect tens of millions of devices transmitting data at an unprecedented scale over limited and often shared radio resources. This complex resource allocation problem is further complicated by several other factors. First, the device density may vary significantly across different geographical areas, creating the so called hot-spot problem. These hot-spots will put the LPWA base stations to a stress test. Second, cross-technology interference can severely degrade the performance of LPWA technologies. This problem is definitely more severe for LPWA technologies operating in the license-exempt and shared ISM bands. Even licensed cellular LPWA technologies operating in-band with broadband services (like voice and video) are equally at this risk. It is not difficult to imagine a scenario when multiple UNB channels of a LPWA technology are simultaneously interfered by a single broadband signal. Further, most LPWA technologies use simple ALOHA or CSMA based MAC protocols, which do not scale well with number of connected devices [75].

Multiple recent studies [36]-[38] investigate if LPWA technologies will be able to support large number of end-devices expected in future city-scale and nationwide deployments. At the time of writing only a few studies are present for LORAWAN. Bor et al. [37] estimate limit on number of nodes that can be supported by a typical LoRAWAN deployment to be 120 per 3.8 ha, a device density far less than expected in urban environments. Georgiou \& Raza [36] further unveil that LORAWAN's coverage probability decay exponentially with number of end devices due to interference. Both studies seem to suggest that end devices should adapt LoRa communication parameters possibly with help from more powerful base stations and exploit base station diversity to overcome this limitation.

Several research directions can be pursued to address the capacity issue of LPWA technologies. These include use of channel diversity, opportunistic spectrum access, and adaptive transmission strategies. Use of channel hopping and multimodem base stations can exploit channel and hardware diversity and is considered already for existing LPWA technologies. Cross-layer solutions can adapt the transmission strategies to the peculiar traffic patterns of LPWA devices and mitigate the effect of cross-technology interference. Further, improvements in existing MAC protocols are required for LPWA technologies to scale them well for a large number of devices transmitting only short messages [75].

In the context of cellular LPWA networks, if excessive IoT/M2M traffic starves the legacy cellular traffic, MNOs may consider deploying LPWA support in unlicensed spectrum. Such an opportunistic use of radio spectrum can benefit from use of cognitive software-defined radios (SDR). SDRs could come in handy when multiple technologies need to compete for shared spectrum.

To cater to areas with a higher device density, LPWA access networks can borrow densification techniques from cellular domain. However, peculiarities of LPWA technologies such as their specialized modulation techniques, strong link asymmetry and mostly uncoordinated operation of end devices pose serious challenges to keep interference levels low in dense deployments.

\section{B. Interference Control and Mitigation}

In future, the number of connected devices will undergo exponential increase, causing higher levels of interference to each other. The devices operating in the shared ISM bands will undergo unprecedented levels of both cross-technology interference as well as self-interference. Some interference measurement studies [76] already point to a possible negative effect on coverage and capacity of LPWA networks. Furthermore, many LPWA technologies like LoRa and SIGFOX resort to simple ALOHA scheme to grant channel access to the lowpower end-devices. This choice of talking randomly without 
listening to others cannot only deteriorate performance, but also generates higher interference [77]. Further, densification of the base station deployments to accommodate more devices is a major source of interference across LPWA cells and requires careful deployment and design of base stations [78].

In an anarchy of tens of wireless technologies and massive number of devices, all sharing the same channels, interference resilient communication and efficient spectrum sharing [79] are key problems, both at technical and regulatory grounds. As interference varies across frequency, time, and space, devices should adapt their transmission schedules to experience the least interference and the best reliability. PHY and MAC layer designs exploiting this diversity at such a large scale need further investigation. Regulatory authorities may also need to step forward to propose rules to enable efficient sharing and cooperation between different wireless technologies in the unlicensed bands [79].

\section{High data-rate modulation techniques}

The LPWA technologies compromise on data rates to reach long distances. Some technologies especially those using UNB modulation in the shared ISM bands offer very low data rates and short payload sizes, limiting their potential business use cases. To support bandwidth hungry use cases, it is meaningful to implement multiple modulation schemes for devices. As per application needs, devices can switch between different modulation schemes so to enable high energy efficiency, long range and high data rate simultaneously.

To achieve this, there is a need for flexible and inexpensive hardware design that can support multiple physical layers, each of which can offer complementary trade-offs to match the range and data rate requirements of applications.

\section{Interoperability between different LPWA technologies}

Given that market is heading towards an intense competition between different LPWA technologies, it is safe to assume that several may coexist in future. Interoperability between these heterogeneous technologies is thus crucial to their longterm profitability. With little to no support for interoperability between different technologies, a need for standards that glue them together is strong. Some of the standardization efforts across ETSI, IEEE, 3GPP, and IETF discussed in Section IV will look into these interoperability issues.

However, for a complete interoperability, several directions should be explored. Firstly, IP can already connect short-range wireless devices using mesh networking. The peculiarities of LPWA technologies limit a direct implementation of the same IP stack on LPWA devices. Alternative solutions based on gateways or backend based solutions are viable candidates. However, all such solutions should scale well with number of devices without degrading performance. Secondly, use of IoT middleware and virtualization techniques can play a major role in connecting LPWA devices. IoT middleware can support multiple radio access technologies and thus make integration of LPWA technologies with rest of IoT technologies straightforward. These middleware can also consolidate data from multiple sources to offer knowledge based value-added services to end-users.

Interoperability is a still an open challenge. Testbeds and open-source tool chains for LPWA technologies are not yet widely available to evaluate interoperability mechanisms.

\section{E. Localization}

LPWA networks expect to generate significant revenue from logistics, supply chain management, and personal IoT applications, where location of mobile objects, vehicles, humans, and animals may be of utmost interest. An accurate localization support is thus an important feature for keeping track of valuables, kids, elderly, pets, shipments, vehicle fleets, etc. In fact, it is regarded as an important feature to enable new applications.

Localization of mobile devices is typically achieved by properties of received signals [80] and time of flight based measurement. All such techniques require very accurate time synchronization and sufficient deployment density of base stations. This is rather easily achieved with a careful network deployment and planning. However, a very limited channel bandwidth of LPWA technologies and an often absence of a direct path between end devices and base stations introduce very large localization error [81], [82]. Thus, doing accurate localization using LPWA transceivers alone is a real challenge.

LPWA networks require new techniques that not only exploit physical layer properties [80] but also combine other established localization techniques to ascertain that accuracy is good enough for real tracking applications.

\section{F. Link optimizations and adaptability}

If a LPWA technology permits, each individual link should be optimized for high link quality and low energy consumption to maximize overall network capacity. Every LPWA technology allows multiple link level configurations that introduce tradeoffs between different performance metrics such as data rate, time-on-air, area coverage, etc. This motivates a need for adaptive techniques that can monitor link quality and then readjust its parameters for better performance.

However for such techniques to work, a feedback from gateway to end devices is usually required over downlink. Link asymmetry that causes downlink of many LPWA technologies (e.g., SIGFOX) to have a lower capacity than uplink is a major hurdle in this case and thus, needs to be addressed in some way.

\section{G. LPWA testbeds and tools}

LPWA technologies enable several smart city applications. A few smart city testbeds e.g. SmartSantander [83] have emerged in recent years. Such testbeds incorporate sensors equipped with different wireless technologies such as $\mathrm{Wi}-\mathrm{Fi}$, IEEE 802.15.4 based networks and cellular networks. However, there are so far no open testbeds for LPWA networks. Therefore, it is not cost-effective to widely design LPWA systems and compare their performance at a metropolitan scale. At the time of writing, only a handful of empirical 
studies [84] compare two our more LPWA technologies under same conditions. In our opinion, it is a significant barrier to entry for potential customers. Providing LPWA technologies as a scientific instrumentation for general public through city governments can act as a confidence building measure. In the meanwhile, analytical models [36], [77] and simulators [85], [86] have recently been proposed for the popular LPWA technologies.

\section{H. Authentication, Security, and Privacy}

Authentication, security, and privacy are some of the most important features of any communication system. Cellular networks provide proven authentication, security, and privacy mechanisms. Use of Subscriber Identity Modules (SIM) simplifies identification and authentication of the cellular devices. LPWA technologies, due to their cost and energy considerations, not only settle for simpler communication protocols but also depart from SIM based authentication. Techniques and protocols are thus required to provide equivalent or better authentication support for LPWA technologies. Further to assure that end devices are not exposed to any security risks over prolonged duration, a support for over-the-air (OTA) updates is a crucial feature. A lack of adequate support for OTA updates poses a great security risk to most LPWA technologies.

Margelis et al. [87] highlight a few security vulnerabilities of the three prominent LPWA technologies namely SIGFOX, LORAWAN, and INGENU. To offer an example, end devices in SIGFOX and LORAWAN networks do not encrypt application payload and the network join request respectively [87], potentially leading to eavesdropping. Further most LPWA technologies use symmetric key cryptography in which the end devices and the networks share a same secret key. Robust and low-power mechanisms for authentication, security, and privacy need further investigation.

\section{Mobility and Roaming}

Roaming of devices between different network operators is a vital feature responsible for the commercial success of cellular networks. Whilst some LPWA technologies do not have the notion of roaming (work on a global scale such as SigFoX), there are others that do not have support for roaming as of the time of this writing. The major challenge is to provide roaming without compromising the lifetime of the devices. To this effect, the roaming support should put minimal burden on the battery powered end-devices. Because the end-devices duty cycle aggressively, it is reasonable to assume that the low power devices cannot receive downlink traffic at all times. Data exchanges over the uplink should be exploited more aggressively. Network assignment is to be resolved in backend systems as opposed to the access network. All the issues related to agility of roaming process and efficient resource management have to be addressed.

Further billing and revenue sharing models for roaming across different networks have to be agreed upon.

International roaming across regions controlled by different spectrum regulations (e.g., USA, Europe or China) is even more challenging. In order to comply to varying spectrum regulations, end devices should be equipped with capabilities to detect the region first and then adhere to the appropriate regional requirements when transmitting data. This adds complexity to end devices and therefore the cost. Simple low cost design to support international roaming is thus required.

\section{J. Support for Service Level Agreements}

The ability to offer certain QoS guarantees can be a competitive differentiator between different LPWA operators. While it is relatively easy to offer QoS guarantees in the licensed spectrum, most proprietary technologies opt for the licenseexempt spectrum for a faster time to market. As a result, they have to adhere to regional regulations on the use of shared spectrum, which may limit the radio duty cycle and transmitted RF power. Cross-technology interference also influences the performance of LPWA technologies.

Providing carrier grade performance on a spectrum shared across multiple uncoordinated technologies and tens of thousands of devices per base station is a significant challenge. Service Level Agreements (SLAs) are likely to be violated due to the factors outside the control of network operators. Therefore, the support for SLAs is expected to be limited in license-exempt bands. Studying such extremely noisy environments to know if some relaxed statistical service guarantees can be provided is a good potential research direction.

\section{K. Co-existence of LPWA technologies with other wireless networks}

Each application has a unique set of requirements, which may vary over different time scales and contexts. If connectivity of the end-devices is supplemented with LPWA technologies in addition to the cellular or wireless LANs, operation of applications can be optimized. Conflicting goals like energy efficiency, high throughput, ultra-low latency and wide area coverage can be achieved by leveraging the benefits of each technology [88], [89]. System-level research is needed to explore benefits of such opportunistic and contextual network access.

There can be different use cases where multiple technologies can cooperate with each other. The ETSI LTN specification [66] lists a few of these use cases for cellular/LPWA cooperation. To offer an example, when cellular connectivity is not available, LPWA technologies can still be used as a fall-back option for sending only low data rate critical traffic. Further, the periodic keep-alive messages of cellular networks can be delegated to energy-efficient LPWA networks [66]. There can be other novel ways for cooperation between LPWA and cellular networks. For instance, LPWA technologies can assist route formation for the device-to-device communication in cellular networks. When some devices outside the cellular coverage need to build a multi-hop route to reach cellular infrastructure, LPWA connectivity can assist in detecting proximity to other serviced devices. These use-cases may have a strong appeal for public safety applications. Further, as we know, LPWA technologies are designed specifically for ultra low data rates. A need of occasionally sending large traffic 
volumes can be met with a complementary cellular connection, which can be activated only on demand.

A joint ownership of LPWA and cellular networks combined with a drop in prices of LPWA devices and connectivity make a strong business case for the above-mentioned use cases. However, there is a need to overcome many systems related challenges.

\section{Support for Data Analytics}

Compared to a human subscriber, the average revenue generated by a single connected M2M/IoT device is rather small. Therefore, network operators see a clear incentive in extending their business beyond the pure connectivity for sake of a higher profitability. One way to do so is by augmenting LPWA networks with sophisticated data analytics support that can convert the raw collected data into contextually relevant information for the end-users. Such knowledge can support end users in making intelligent decisions, earning higher profits, or bringing their operational costs down. Network operators thus can monetize this by selling knowledge to end users.

There are however enormous challenges associated with providing a LPWA network as a service to the end-users. It requires a unified management of business platform and a scalable integration with the cloud. One of the main challenges is also to offer custom-tailored services to many different vertical industries, effectively covering different use cases ideally by a single LPWA technology.

\section{BUSINESS CONSIDERATIONS}

With the dawn of the M2M communications paradigm, 2G seemed to be a reasonable fit for catering to the requirements of these applications. Given the spectrum scarcity worldwide and the high capital expenditure incurred in acquiring new spectrum, operators appear to be in a dilemma whether to continue using $2 \mathrm{G}$ systems for serving M2M customers or re-farm the spectrum making way for new technologies such as LTE and its variants. Announcements from a handful of operators to transition to the latter created a hole in the market. Since then, several new LPWA technologies such as those mentioned in Table III have been aggressively trying to fill this gap with the hope of staking their claim to the pole position. Only those technologies have been included in the table for which substantial information is available in the public domain, those that have a wide variety of products already available in the market and those that have had large scale deployments. The much anticipated NB-IoT standard from the cellular world has been included to provide a perspective as to how the different forerunners in the market stack up against a potential cellular offering in the making.

It is worth emphasizing that there is no one size fits all solution with each of these approaches having their pros and cons as highlighted in the table. The market is still up for grabs and players have several strategic options to consider depending on their circumstances, e.g., those needing to deploy an IoT solution immediately will have to hedge their bets on LoRa, SigFox, INGENU, WeightLess-N etc. whereas others can afford to wait until the 3GPP finalizes standards such as NB-IoT which is still work-in-progress. In the meanwhile, the cellular operators themselves seem to have hedged their bets on LORa and SIGFOX with several operators making big investments in one or the other. In any case, it looks like a win-win situation for the operators irrespective of how the situation plays out since these technologies could play a complementary role to the potential NB-IoT standard that is currently being baked. Also, the fact that operators have invested in these technologies reduces the uncertainty from a longevity perspective ${ }^{1}$ for the adopters of these solutions.

It is envisaged that LoRa, SIGFox, and INGENU will continue to challenge the hegemony of the cellular players and all four are likely to share the pie in the long run. It is expected that there would be a varying degree of adoption across multiple market segments and pricing models [91] are likely to have a significant impact on the success of different technologies.

In a nutshell, as of this writing fierce battles continue to be fought to capture the LPWA market share and competitors are leaving no stone unturned to attack each other's propositions (see [92]-[94]).

\section{CONCLUSION}

Wide area coverage, low power consumption, and inexpensive wireless connectivity blends together in LPWA technologies to enable a strong business case for low throughput IoT/M2M applications that do not require ultra-low latency. However, this combination of often conflicting goals is a result of carefully designed physical and MAC layer techniques, precisely surveyed in this paper. To tap into the huge IoT/M2M market, several commercial providers exploit different innovative techniques in their LPWA connectivity solutions. The variety of these solutions have resulted in a fragmented market, highlighting a dire need for standards. We provided a comprehensive overview of many such standardization efforts led by several SDOs and SIGs. We observe that most standards focus on physical and MAC layers. A gap at the upper layers (application, transport, network etc.) is to be bridged. Further, we point out important challenges that LPWA technologies face today and possible directions to overcome them. We encourage further developments in LPWA technologies to push the envelop of connecting massive number of devices in future.

\section{REFERENCES}

[1] "Cellular networks for massive iot: Enabling low power wide area applications," Ericsson, Tech. Rep., January 2016, ericsson White Paper. [Online]. Available: https://www.ericsson.com/res/docs/ whitepapers/wp_iot.pdf

[2] E. Berthelsen and J. Morrish, "Forecasting the internet of things revenue opportunity," Machina Research, Tech. Rep., April 2015. [Online]. Available: https://machinaresearch.com/report_pdf/313

[3] "Lte evolution for iot connectivity," Nokia, Tech. Rep., 2016, nokia White Paper. [Online]. Available: http://resources.alcatel-lucent.com/ asset/200178

[4] X. Xiong, K. Zheng, R. Xu, W. Xiang, and P. Chatzimisios, "Low power wide area machine-to-machine networks: key techniques and prototype," IEEE Communications Magazine, vol. 53, no. 9, pp. 64-71, September 2015 .

\footnotetext{
${ }^{1}$ Recall the nightmares that the announcements regarding sunset of the $2 \mathrm{G}$ systems might have given to the M2M customers
} 
TABLE III

BUSINESS CONSIDERATIONS FOR VARIOUS LPWA TECHNOLOGIES (?=NOT KNOWN)

\begin{tabular}{|c|c|c|c|c|c|}
\hline & SIGFOX $[\overline{9}]$ & LORAWAN 15 & WEIGHTLESS-N & INGENU $[\overline{90}]$ & 3GPP Cellular IoT \\
\hline Deployment model & $\begin{array}{l}\text { Nationwide (multiple } \\
\text { countries) }\end{array}$ & $\begin{array}{c}\text { Private or nationwide } \\
\text { networks }\end{array}$ & Private networks & $\begin{array}{c}\text { Private or nationwide } \\
\text { networks }\end{array}$ & Nationwide networks \\
\hline Ease of roaming & $\begin{array}{l}\text { Seamless roaming across } \\
\text { SIGFox networks in } \\
\text { different countries at no } \\
\text { extra charges }\end{array}$ & $\begin{array}{l}\text { Roaming agreements } \\
\text { required }\end{array}$ & Not applicable & $?$ & $\begin{array}{l}\text { Operator alliances for } \\
\text { cross-border roaming }\end{array}$ \\
\hline SLA support & $x$ & $x$ & $x$ & $x$ & $\checkmark$ \\
\hline Device availability & $\checkmark$ & $\checkmark$ & Focus is on gateway & $\checkmark$ & $\begin{array}{c}\times \text { (Still in standardization } \\
\text { phase, devices will emerge } \\
\text { later })\end{array}$ \\
\hline $\begin{array}{l}\text { Over-the-air } \\
\text { updates for } \\
\text { devices }\end{array}$ & $x$ & possible & $x$ & possible & likely be made available \\
\hline $\begin{array}{c}\text { Supplier } \\
\text { ecosystem }\end{array}$ & $\begin{array}{l}\text { Transceivers and modules } \\
\text { from many vendors }\end{array}$ & $\begin{array}{l}\text { Limited choice of vendors } \\
\text { for transceivers, several } \\
\text { module vendors }\end{array}$ & Limited choice of vendors & $\begin{array}{l}\text { Transceivers and modules } \\
\text { from many vendors }\end{array}$ & $\begin{array}{l}\text { Availability likely from all } \\
\text { the usual vendors once } \\
\text { standard is ratified }\end{array}$ \\
\hline Licensing & $\begin{array}{c}\text { Technology freely } \\
\text { available for chip/device } \\
\text { vendors. Network } \\
\text { operators pay royalty to } \\
\text { SIGFOX (revenue sharing } \\
\text { basis) }\end{array}$ & $\begin{array}{l}\text { Technology licensed by } \\
\text { device vendors. No } \\
\text { royalty to be paid by } \\
\text { network operators }\end{array}$ & $\begin{array}{c}\text { Technology freely } \\
\text { available for chip/device } \\
\text { vendors. No royalty } \\
\text { thereafter. }\end{array}$ & $\begin{array}{c}\text { Upfront fee }+ \text { per } \\
\text { application \& per device } \\
\text { fee / year (No revenue } \\
\text { sharing) }\end{array}$ & $\begin{array}{l}\text { Standardized technology. } \\
\text { Usual cellular model } \\
\text { likely to prevail }\end{array}$ \\
\hline Deployment status & $\begin{array}{c}\text { Network deployed \& } \\
\text { running in several } \\
\text { countries. Several } \\
\text { operators have invested in } \\
\text { SIGFox }\end{array}$ & $\begin{array}{c}\text { Early trials \& } \\
\text { deployments by some } \\
\text { operators. Several } \\
\text { operators are members of } \\
\text { LoRa }{ }^{\mathrm{TM}} \text { Alliance }\end{array}$ & $\begin{array}{l}\text { Some trials but no major } \\
\text { deployments }\end{array}$ & $\begin{array}{c}\text { Several private } \\
\text { deployments in over } 5 \\
\text { continents }\end{array}$ & $\begin{array}{l}\text { Early days with some } \\
\text { in-house trials with } \\
\text { pre-standardized } \\
\text { technology by handful of } \\
\text { operators }\end{array}$ \\
\hline $\begin{array}{l}\text { Longevity offered } \\
\text { by the solution }\end{array}$ & $\begin{array}{l}\text { Deployments in several } \\
\text { countries. Not much } \\
\text { insight into transition plan } \\
\text { should SNOs find it } \\
\text { infeasible to run the } \\
\text { network. Transitioning } \\
\text { will entail replacement of } \\
\text { endpoint/communications } \\
\text { module in the endpoints. }\end{array}$ & $\begin{array}{l}\text { Some deployments by } \\
\text { cellular operators in a few } \\
\text { countries. No insight into } \\
\text { transition plan should } \\
\text { LoRa network be } \\
\text { decommissioned. } \\
\text { Transitioning will entail } \\
\text { replacement of } \\
\text { endpoint/communications } \\
\text { module in the endpoints. }\end{array}$ & $\begin{array}{l}\text { No deployments so far so } \\
\text { longevity is questionable }\end{array}$ & $\begin{array}{l}\text { Deployments in several } \\
\text { countries. Not much } \\
\text { insight into transition plan } \\
\text { should MNOs find it } \\
\text { infeasible to run the } \\
\text { network. Transitioning } \\
\text { will entail replacement of } \\
\text { endpoint/communications } \\
\text { module in the endpoints. }\end{array}$ & $\begin{array}{l}\text { Promising as this being a } \\
\text { solution designed } \\
\text { exclusively for IoT, is less } \\
\text { likely to be } \\
\text { de-commissioned. }\end{array}$ \\
\hline
\end{tabular}

[5] S. Wilson. The future of $3 \mathrm{~g}$ : the case for decommissioning. [Online]. Available: http://www.analysysmason.com/3G-decommission-Oct2015

[6] J. Petajajarvi, K. Mikhaylov, A. Roivainen, T. Hanninen, and M. Pettissalo, "On the coverage of lpwans: range evaluation and channel attenuation model for lora technology," in ITS Telecommunications (ITST), 2015 14th International Conference on, Dec 2015, pp. 55-59.

[7] "5g radio access," Ericsson, Tech. Rep., April 2016, ericsson White Paper. [Online]. Available: https://www.ericsson.com/res/docs/ whitepapers/wp-5g.pdf

[8] F. Adelantado, X. Vilajosana, P. Tuset-Peiro, B. Martinez, and J. Melia, "Understanding the limits of lorawan," arXiv preprint arXiv:1607.08011, 2016.

[9] Sigfox. [Online]. Available: http://www.sigfox.com/

[10] N. Sornin, M. Luis, T. Eirich, and T. Kramp, "Lorawan specification," LoRa Alliance, Tech. Rep., 2015. [Online]. Available: https://www.lora-alliance.org/portals/0/specs/LoRaWAN\% 20Specification\%201R0.pdf

[11] European telecommunications standards institute. [Online]. Available: http://www.etsi.org/

[12] The 3rd generation partnership project. [Online]. Available: http: //www.3gpp.org/

[13] Institute of electrical and electronics engineers. [Online]. Available: https://www.ieee.org/

[14] Internet engineering task force (ietf). [Online]. Available: https: //www.ietf.org/

[15] Lora alliance. [Online]. Available: https://www.lora-alliance.org/

[16] Weightless. [Online]. Available: http://www.weightless.org/

[17] Dash7 alliance. [Online]. Available: http://www.dash7-alliance.org/

[18] "Rpma technology for the internet of things," Ingenu, Tech. Rep., 2016. [Online]. Available: http://theinternetofthings.report/ Resources/Whitepapers/4cbc5e5e-6ef8-4455-b8cd-f6e3888624cb_ RPMA\%20Technology.pdf

[19] P. Massam, P. Bowden, and T. Howe, "Narrow band transceiver," Jan. 9 2013, eP Patent 2,092,682. [Online]. Available: http: //www.google.com/patents/EP2092682B1?cl=pt-PT

[20] G. Strazdins, A. Elsts, K. Nesenbergs, and L. Selavo, "Wireless sensor network operating system design rules based on real-world deployment survey," Journal of Sensor and Actuator Networks, vol. 2, no. 3, pp. 509-556, 2013. [Online]. Available: http: //www.mdpi.com/2224-2708/2/3/509
[21] F. J. Oppermann, C. A. Boano, and K. Römer, A Decade of Wireless Sensing Applications: Survey and Taxonomy. Berlin, Heidelberg: Springer Berlin Heidelberg, 2014, pp. 11-50. [Online]. Available: http://dx.doi.org/10.1007/978-3-642-40009-4_2

[22] I. Demirkol, C. Ersoy, F. Alagoz et al., "Mac protocols for wireless sensor networks: a survey," IEEE Communications Magazine, vol. 44, no. 4, pp. 115-121, 2006.

[23] G. Anastasi, M. Conti, M. Di Francesco, and A. Passarella, "Energy conservation in wireless sensor networks: A survey," Ad hoc networks, vol. 7, no. 3, pp. 537-568, 2009.

[24] P. M. John Burns, Selcuk Kirtay, "Future use of licence exempt radio spectrum," Plum Consulting, Tech. Rep., 2015. [Online]. Available: http://www.plumconsulting.co.uk/pdfs/Plum_July_ 2015_Future_use_of_Licence_Exempt_Radio_Spectrum.pdf

[25] V. Raghunathan, S. Ganeriwal, and M. Srivastava, "Emerging techniques for long lived wireless sensor networks," IEEE Communications Magazine, vol. 44, no. 4, pp. 108-114, April 2006.

[26] U. Raza, A. Bogliolo, V. Freschi, E. Lattanzi, and A. L. Murphy, "A two-prong approach to energy-efficient wsns," Ad Hoc Netw., vol. 45, no. C, pp. 1-12, Jul. 2016. [Online]. Available: http: //dx.doi.org/10.1016/j.adhoc.2016.03.005

[27] U. Raza, "From energy efficient to energy neutral wireless sensor networks," Ph.D. dissertation, University of Trento, 2015.

[28] Z. Xie, R. Xu, and L. Lei, "A study of clear channel assessment performance for low power wide area networks," in Wireless Communications, Networking and Mobile Computing (WiCOM 2014), 10th International Conference on, Sept 2014, pp. 311-315.

[29] K. E. Nolan, M. Y. Kelly, M. Nolan, J. Brady, and W. Guibene, "Techniques for resilient real-world iot," in 2016 International Wireless Communications and Mobile Computing Conference (IWCMC), Sept 2016, pp. 222-226.

[30] U. Raza, A. Camerra, A. L. Murphy, T. Palpanas, and G. P. Picco, "Practical data prediction for real-world wireless sensor networks," IEEE Transactions on Knowledge and Data Engineering, vol. 27, no. 8, pp. 2231-2244, Aug 2015.

[31] Openfog. [Online]. Available: https://www.openfogconsortium.org/

[32] Mobile edge computing. [Online]. Available: http://www.etsi.org/ technologies-clusters/technologies/mobile-edge-computing 
[33] "Nb-iot - enabling new business opportunities," Hawei Technologies Co., Tech. Rep., 2015. [Online]. Available: http://www.huawei.com/ minisite/4-5g/img/NB-IOT.pdf

[34] "Mobile internet of things: low power wide area connectivity," GSMA Mobile IoT, Tech. Rep., 2016, gSMA Industry Paper. [Online]. Available: http://www.gsma.com/connectedliving/wp-content/uploads/2016/03/ Mobile-IoT-Low-Power-Wide-Area-Connectivity-GSMA-Industry-Paper pdf

[35] Sigfox's ecosystem delivers the worlds first ultra-low cost modules to fuel the internet of things mass market deployment. [Online]. Available: https://www.sigfox.com/en/press/ sigfox-s-ecosystem-delivers-world-s-first-ultra-low-cost-modules-to-fuel-in

[36] O. Georgiou and U. Raza, "Low power wide area network analysis: Can lora scale?" IEEE Wireless Communications Letters, vol. PP, no. 99, pp. $1-1,2017$.

[37] M. C. Bor, . Roedig, T. Voigt, and J. M. Alonso, "Do lora low-power wide-area networks scale?" in Proceedings of the 19th ACM International Conference on Modeling, Analysis and Simulation of Wireless and Mobile Systems, ser. MSWiM '16. New York, NY, USA: ACM, 2016, pp. 59-67. [Online]. Available: http: //doi.acm.org/10.1145/2988287.2989163

[38] K. Mikhaylov, . J. Petaejaejaervi, and T. Haenninen, "Analysis of capacity and scalability of the lora low power wide area network technology," in European Wireless 2016; 22th European Wireless Conference, May 2016, pp. 1-6.

[39] Comparison of low-power wan alternatives. [Online]. Available: http://www.cnx-software.com/2015/09/21/ comparison-table-of-low-power-wan-standards-for-industrial-applications/

[40] "Waspmote sigfox networking guide," Libelium Comunicaciones Distribuidas S.L., Tech. Rep., November 2015, document Version: v4.1. [Online]. Available: http://www.libelium.com/downloads/ documentation/sigfox_networking_guide.pdf

[41] F. Sforza, "Communications system," March 2013, uS Patent 8,406,275. [Online]. Available: https://www.google.com/patents/US8406275

[42] Semtech. [Online]. Available: http://www.semtech.com/

[43] B. Reynders, W. Meert, and S. Pollin, "Range and coexistence analysis of long range unlicensed communication," in 2016 23rd International Conference on Telecommunications (ICT), May 2016, pp. 1-6.

[44] K. E. Nolan, W. Guibene, and M. Y. Kelly, "An evaluation of low power wide area network technologies for the internet of things," in 2016 International Wireless Communications and Mobile Computing Conference (IWCMC), Sept 2016, pp. 439-444.

[45] O. Iova, A. Murphy, G. P. Picco, L. Ghiro, D. Molteni, F. Ossi, and F. Cagnacci, "Lora from the city to the mountains: Exploration of hardware and environmental factors," in Proceedings of the 2017 International Conference on Embedded Wireless Systems and Networks, ser. EWSN '17, 2017.

[46] P. Neumann, J. Montavont, and T. Nol, "Indoor deployment of lowpower wide area networks (lpwan): A lorawan case study," in 2016 IEEE 12th International Conference on Wireless and Mobile Computing, Networking and Communications (WiMob), Oct 2016, pp. 1-8.

[47] J. Petjjrvi, K. Mikhaylov, M. Hmlinen, and J. Iinatti, "Evaluation of lora lpwan technology for remote health and wellbeing monitoring," in 2016 10th International Symposium on Medical Information and Communication Technology (ISMICT), March 2016, pp. 1-5.

[48] T. Myers, D. Werner, K. Sinsuan, J. Wilson, S. Reuland, P. Singler, and M. Huovila, "Light monitoring system using a random phase multiple access system," Jul. 2 2013, uS Patent 8,477,830. [Online]. Available: https://www.google.com/patents/US8477830

[49] Telensa. [Online]. Available: http://www.telensa.com/

[50] Talq consortium. [Online]. Available: http://www.talq-consortium.org

[51] W. Guibene, K. E. Nolan, and M. Y. Kelly, "Survey on clean slate cellular-iot standard proposals," in 2015 IEEE International Conference on Computer and Information Technology; Ubiquitous Computing and Communications; Dependable, Autonomic and Secure Computing; Pervasive Intelligence and Computing, Oct 2015, pp. 1596-1599.

[52] "Ieee standard for local and metropolitan area networks-part 15.4: Lowrate wireless personal area networks (lr-wpans)," IEEE Std 802.15.42011 (Revision of IEEE Std 802.15.4-2006), pp. 1-314, Sept 2011.

[53] "Ieee standard for information technology-telecommunications and information exchange between systems local and metropolitan area networks-specific requirements part 11: Wireless lan medium access control (mac) and physical layer (phy) specifications," IEEE Std 802.11 2012 (Revision of IEEE Std 802.11-2007), pp. 1-2793, March 2012.
[54] K. Zheng, S. Zhao, Z. Yang, X. Xiong, and W. Xiang, "Design and implementation of lpwa-based air quality monitoring system," IEEE Access, vol. 4, pp. 3238-3245, 2016.

[55] K.-H. Chang and B. Mason, "The ieee 802.15.4g standard for smart metering utility networks," in Smart Grid Communications (SmartGridComm), 2012 IEEE Third International Conference on, Nov 2012, pp. 476-480.

[56] S. Tozlu, M. Senel, W. Mao, and A. Keshavarzian, "Wi-fi enabled sensors for internet of things: A practical approach," IEEE Communications Magazine, vol. 50, no. 6, pp. 134-143, June 2012.

[57] Ieee p802.11- task group ah. [Online]. Available: http://www.ieee802. org/11/Reports/tgah_update.htm

IfroshetF.ofAthaimgs. A. Bel, B. Bellalta, J. Barcelo, and M. Oliver, "Ieee 802.11ah: the wifi approach for $\mathrm{m} 2 \mathrm{~m}$ communications," IEEE Wireless Communications, vol. 21, no. 6, pp. 144-152, December 2014.

[59] S. Andreev, O. Galinina, A. Pyattaev, M. Gerasimenko, T. Tirronen, J. Torsner, J. Sachs, M. Dohler, and Y. Koucheryavy, "Understanding the iot connectivity landscape: a contemporary $\mathrm{m} 2 \mathrm{~m}$ radio technology roadmap," IEEE Communications Magazine, vol. 53, no. 9, pp. 32-40, 2015.

[60] M. R. Palattella, M. Dohler, A. Grieco, G. Rizzo, J. Torsner, T. Engel, and L. Ladid, "Internet of things in the 5g era: Enablers, architecture, and business models," IEEE Journal on Selected Areas in Communications, vol. 34, no. 3, pp. 510-527, March 2016.

[61] A. Minaburo, L. Toutain, C. Gomez, J. Paradells, and J. Crowcroft, "LPWAN Survey and GAP Analysis," Internet Engineering Task Force, Internet-Draft draft-minaburo-lpwan-gap-analysis-02, Oct. 2016, work in Progress. [Online]. Available: https://tools.ietf.org/html/ draft-minaburo-lpwan-gap-analysis-02

62] Low-power wide area networks (lpwan). [Online]. Available: https: //datatracker.ietf.org/wg/lpwan/charter/

[63] A. Hazmi, J. Rinne, and M. Valkama, "Feasibility study of i 802.11ah radio technology for iot and $\mathrm{m} 2 \mathrm{~m}$ use cases," in 2012 IEEE Globecom Workshops, Dec 2012, pp. 1687-1692.

[64] Ieee p802.11 - lrlp topic interest group. [Online]. Available: http://www.ieee802.org/11/Reports/lrlp_update.htm

[65] T. G. et al., "Long range low power (lrlp) operation in 802.11: Use cases and functional requirements: Guidelines for par development," IEEE P802.11 Wireless LANs, February 2016.

[66] "Low throughput networks (ltn); use cases for low throughput networks," ETSI GS LTN 001 V1.1.1, September 2014. [Online]. Available: http://www.etsi.org/deliver/etsi_gs/LTN/001_099/001/01.01. 01_60/gs_LTN001v010101p.pdf

[67] "Low throughput networks (1tn); functional architecture," ETSI GS LTN 002 V1.1.1, September 2014. [Online]. Available: http://www.etsi.org/deliver/etsi_gs/LTN/001_099/002/01.01.01_60/ gs_LTN002v010101p.pdf

[68] "Low throughput networks (ltn);protocols and interfaces," ETSI GS LTN 003 V1.1.1, September 2014. [Online]. Available: http://www.etsi.org/deliver/etsi_gs/LTN/001_099/003/01.01. 01_60/gs_LTN003v010101p.pdf

[69] D. Flore, "3gpp standards for the internet-of-things," February 2016 , gSMA MIoT. [Online]. Available: http://www.3gpp.org/news-events/ 3gpp-news/1766-iot_progress

[70] Y.-P. E. Wang, X. Lin, A. Adhikary, A. Grövlen, Y. Sui, Y. Blankenship, J. Bergman, and H. S. Razaghi, "A primer on 3gpp narrowband internet of things (nb-iot)," arXiv preprint arXiv:1606.04171, 2016.

[71] "7 nb-iot surprises you need to know about," Ingenu, Tech. Rep., October 2016. [Online]. Available: http://www.ingenu.com/portfolio/ seven-nb-iot-surprises-you-need-to-know-about/

[72] A. Pelov, L. Toutain, and Y. Delibie, "Constrained Signaling Over LP-WAN," Internet Engineering Task Force, Internet-Draft draft-pelovcore-cosol-01, Feb. 2016, work in Progress. [Online]. Available: https://tools.ietf.org/html/draft-pelov-core-cosol-01

[73] M. Weyn, G. Ergeerts, R. Berkvens, B. Wojciechowski, and Y. Tabakov, "Dash7 alliance protocol 1.0: Low-power, mid-range sensor and actuator communication," in Standards for Communications and Networking (CSCN), 2015 IEEE Conference on, Oct 2015, pp. 54-59.

[74] "Information technology - radio frequency identification for item management - part 7: Parameters for active air interface communications at 433 mhz," ISO/IEC 18000-7:2009, August 2009.

[75] A. Laya, C. Kalalas, F. Vazquez-Gallego, L. Alonso, and J. AlonsoZarate, "Goodbye, aloha!" IEEE Access, vol. PP, no. 99, pp. 1-1, 2016.

[76] M. Lauridsen, B. Vejlgaard, I. Kovács, H. C. Nguyen, and P. E. Mogensen, "Interference measurements in the european $868 \mathrm{mhz}$ ism band with focus on lora and sigfox," in Ieee Wireless Communications and Networking Conference 2017, 2017. 
[77] Y. Mo, C. Goursaud, and J. M. Gorce, "Theoretical analysis of unb-based iot networks with path loss and random spectrum access," in 2016 IEEE 27th Annual International Symposium on Personal, Indoor, and Mobile Radio Communications (PIMRC), Sept 2016, pp. 1-6.

[78] T. Voigt, M. Bor, U. Roedig, and J. Alonso, "Mitigating inter-network interference in lora networks," in Proceedings of the 2017 International Conference on Embedded Wireless Systems and Networks, ser. EWSN '17, 2017.

[79] F. Beltran, S. K. Ray, and J. A. Gutirrez, "Understanding the current operation and future roles of wireless networks: Co-existence, competition and co-operation in the unlicensed spectrum bands," IEEE Journal on Selected Areas in Communications, vol. 34, no. 11, pp. 2829-2837, Nov 2016.

[80] A. Zanella, "Best practice in rss measurements and ranging," IEEE Communications Surveys Tutorials, vol. PP, no. 99, pp. 1-1, 2016.

[81] K. J. Krizman, T. E. Biedka, and S. Rappaport, "Wireless position location: fundamentals, implementation strategies, and sources of error," in IEEE Vehicular Technology Conference, vol. 47. INSTITUTE OF ELECTRICAL ENGINEERS INC (IEEE), 1997, pp. 919-923.

[82] R. Henriksson, "Indoor positioning in lorawan networks: Evaluation of rss positioning in lorawan networks using commercially available hardware," Master's thesis, Chalmers University of Technology, 2016.

[83] L. Sanchez, L. Muoz, J. A. Galache, P. Sotres, J. R. Santana, V. Gutierrez, R. Ramdhany, A. Gluhak, S. Krco, E. Theodoridis, and D. Pfisterer, "Smartsantander: Iot experimentation over a smart city testbed," Computer Networks, vol. 61, pp. 217 - 238, 2014, special issue on Future Internet Testbeds Part I. [Online]. Available: http://www.sciencedirect.com/science/article/pii/S1389128613004337

[84] S. Kartakis, B. D. Choudhary, A. D. Gluhak, L. Lambrinos, and J. A. McCann, "Demystifying low-power wide-area communications for city iot applications," in Proceedings of the Tenth ACM International
Workshop on Wireless Network Testbeds, Experimental Evaluation, and Characterization, ser. WiNTECH '16. New York, NY, USA: ACM, 2016, pp. 2-8. [Online]. Available: http://doi.acm.org/10.1145/2980159. 2980162

[85] Lorasim. [Online]. Available: http://www.lancaster.ac.uk/scc/sites/lora/ lorasim.html

[86] D. Magrin, "Network level performances of a lora system," Master's thesis, University of Padova, 2016.

[87] G. Margelis, R. Piechocki, D. Kaleshi, and P. Thomas, "Low throughput networks for the iot: Lessons learned from industrial implementations," in Internet of Things (WF-IoT), 2015 IEEE 2nd World Forum on, Dec 2015, pp. 181-186.

[88] M. Taneja, "802.11ah - lpwa interworking," in 2016 IEEE NetSoft Conference and Workshops (NetSoft), June 2016, pp. 441-446.

[89] — , "Lte-lpwa networks for iot applications," in 2016 International Conference on Information and Communication Technology Convergence (ICTC), Oct 2016, pp. 396-399.

[90] I. Morris, "Ingenu revs up iot rhetoric," May 2016. [Online]. Available: http://goo.gl/S2ySYg

[91] A. Davies, "On lpwans: Why sigfox and lora are rather different, and the importance of the business model," March 2015. [Online]. Available: http://goo.gl/Hkvm0Z

[92] Lora alliance defends tech against sigfox slur. [Online]. Available: http://www.lightreading.com/iot/iot-strategies/ lora-alliance-defends-tech-against-sigfox-slur/d/d-id/722982

[93] Vodafone to 'crush' lora, sigfox with nbiot. [Online]. Available: http://www.lightreading.com/iot/ vodafone-to-crush-lora-sigfox-with-nb-iot/d/d-id/722882

[94] Ingenu revs up iot rhetoric. [Online]. Available: http://www.lightreading. com/iot/iot-strategies/ingenu-revs-up-iot-rhetoric/d/d-id/723284 